\title{
Complex matrix model duality
}

\author{
T. W. Brown* \\ DESY, Hamburg, Theory Group, Notkestrasse, 85, D-22603 Hamburg, Germany
}

(Received 11 January 2011; published 1 April 2011)

\begin{abstract}
The same complex matrix model calculates both tachyon scattering for the $c=1$ noncritical string at the self-dual radius and certain correlation functions of operators which preserve half the supersymmetry in $\mathcal{N}=4$ super-Yang-Mills theory. It is dual to another complex matrix model where the couplings of the first model are encoded in the Kontsevich-like variables of the second. The duality between the theories is mirrored by the duality of their Feynman diagrams. Analogously to the Hermitian Kontsevich-Penner model, the correlation functions of the second model can be written as sums over discrete points in subspaces of the moduli space of punctured Riemann surfaces.
\end{abstract}

DOI: $10.1103 /$ PhysRevD.83.085002

PACS numbers: 11.15.Pg, 04.60.Kz, 11.25.Tq

\section{INTRODUCTION}

In recent examples of gauge-gravity duality, Feynman graphs of the gauge theory are lifted to open string diagrams whose world sheet holes are summed over, replacing them with closed string insertions, to give a closed string theory in a different background. Open-closed string dualities like this, such as the three-dimensional Chern-Simons to conifold duality $[1,2]$ or the Kontsevich matrix model to two-dimensional (2D) topological gravity duality [3,4], have been categorized by Gopakumar as of the " $F$ type" [5] because it is the faces of Feynman graphs which are replaced by closed string insertions. On the other hand, dualities such as that between four-dimensional (4D) $\mathcal{N}=4$ super Yang-Mills (SYM) and type IIB closed string theory on $\operatorname{AdS}_{5} \times S^{5}$ [6] are of the " $V$ type" [5] because it is the vertices of the Feynman diagrams, corresponding to local operators and interaction vertices, which are replaced by closed string insertions. All known openclosed dualities are either of the $F$ type or the $V$ type [5]. The possibility was raised by Gopakumar [5] that for every gauge-gravity duality the closed string theory has open string duals of both types, related to each other by graph duality. Topological gravity in 2D was given as an example, with the theory of the $V$ type being the doublescaled Hermitian matrix model [7-9] and that of the $F$ type being the Kontsevich Hermitian matrix model [3]. Using the proof of equivalence in [10], the graph duality can be shown dynamically by integrating in and out different fields, so that at different steps vertices are replaced by faces and vice versa [5].

In this paper we show this open-open duality between a complex matrix model of the $V$ type called the $Z$ model and another complex matrix model of the $F$ type called the $F$ model. The $Z$ model is known to calculate certain correlation functions of half-BPS operators in $\mathcal{N}=4$ super Yang-Mills as well as tachyon scattering amplitudes for the $c=1$ string at the self-dual radius. The dual $F$ model

*nworbmot@gmail.com provides a new Kontsevich-Penner matrix model for these amplitudes and thus throws new light on how to write them as integrals over the moduli space of Riemann surfaces.

The $Z$ model is

$$
Z(\{t\},\{\bar{t}\})=\int[d Z]_{N \times N}^{\mathbb{C}} e^{-\operatorname{tr}\left(Z Z^{\dagger}\right)+\sum_{k=1}^{\infty} t_{k} \operatorname{tr}\left(Z^{k}\right)+\sum_{k=1}^{\infty} \bar{t}_{k} \operatorname{tr}\left(Z^{\dagger k}\right)} .
$$

It has two infinite sets of couplings which are often called times in the literature because of the relation with the $\tau$ function of the Toda integrable hierarchy.

From the $4 \mathrm{D} \mathcal{N}=4$ super Yang-Mills perspective, the $Z$ model is a generating function for certain correlation functions of holomorphic and antiholomorphic half-BPS operators built from a single complex scalar transforming in the adjoint of the gauge group $U(N)[11,12]$. In "extremal" correlation functions, for which the antiholomorphic operators are all at the same spacetime position, the spacetime dependence of the correlation function factors out of the result; the $Z$ model computes the remaining combinatorial factor, which is an expansion in $1 / N .^{1}$ Because local operators in $\mathcal{N}=4$ super Yang-Mills (vertices in the $Z$ model) map to string (or supergravity in this case) states, the $Z$ model is of the $V$ type.

In the guise of a normal matrix model [14] the $Z$ model is also a generating function for the correlation functions of integer-momentum massless tachyons in the $c=1$ noncritical string compactified at the self-dual radius. The cosmological constant $\mu$ of the $c=1$ string, which controls the genus expansion, is related to the rank $N$ of the complex matrix by $N=-i \mu$. In contrast to the

\footnotetext{
${ }^{1}$ This combinatorial factor is unchanged if all the holomorphic operators are also taken to the same spacetime position, so really the $Z$ model just generates the two-point function of multitrace half-BPS states. This is like a metric on the multitrace states, cf. the discussion in [13]. Note too that the extremal correlation functions are known not to renormalize when the coupling is nontrivial.
} 
double-scaled matrix quantum mechanics (MQM) for the $c=1$ string, $^{2}$ the $Z$ model requires no scaling limit and works at finite $N$. This is reflected in the fact that the $Z$ model is not a triangulation of the Riemann surface itself but rather, through its dual, a triangulation of the moduli space of punctured Riemann surfaces. This relation between the half-BPS sector of the $\mathrm{AdS}_{5}$ duality and the $c=1$ string has been explored in $[17,18]$ based on the similarity of their MQM descriptions [19] and shown to be exact at the self-dual radius in [20]. This connection is in the spirit of the minimal $(p, 1)$ string embedding in the $\mathrm{AdS}_{3}$ duality [21].

The map from tachyons $\mathcal{T}_{p}$ with integer momentum $p$ to matrix variables is for $k>0$

$$
\mathcal{T}_{k} \rightarrow \operatorname{tr}\left(Z^{k}\right), \quad \mathcal{T}_{-k} \rightarrow \operatorname{tr}\left(Z^{\dagger k}\right) .
$$

The individual tachyon correlation functions are then

$$
\begin{aligned}
& \left\langle\mathcal{T}_{k_{1}} \cdots \mathcal{T}_{k_{p}} \mathcal{T}_{-\bar{k}_{1}} \cdots \mathcal{T}_{-\bar{k}_{q}}\right\rangle_{c=1} \\
& \quad=\left\langle\operatorname{tr}\left(Z^{k_{1}}\right) \cdots \operatorname{tr}\left(Z^{k_{p}}\right) \operatorname{tr}\left(Z^{\dagger \bar{k}_{1}}\right) \cdots \operatorname{tr}\left(Z^{\dagger \bar{k} q}\right)\right\rangle .
\end{aligned}
$$

On the right-hand side, the correlation function is taken using the complex matrix model with Gaussian action $\operatorname{tr}\left(Z Z^{\dagger}\right)$. It is computed by Wick contracting with the propagator

$$
\left\langle Z_{f}^{e} Z_{h}^{\dagger g}\right\rangle=\delta_{h}^{e} \delta_{f}^{g} .
$$

These correlation functions can be computed to all orders in $N$ using symmetric group techniques $[13,19]$.

The dual $F$ model is exactly the same function of $\{t\},\{\bar{t}\}$

$$
\begin{aligned}
& Z(\{t\},\{\vec{t}\})=\int[d F]_{n \times n}^{\mathbb{C}} e^{-\operatorname{tr}\left(F F^{\dagger}\right)-N \operatorname{tr} \log \left(1-A^{-1} F B^{-1} F^{\dagger}\right)} \\
& =\int[d F]_{n \times n}^{\mathbb{C}} e^{-\operatorname{tr}\left(F F^{\dagger}\right)+N} \sum_{k=1}^{\infty}(1 / k) \operatorname{tr}\left[\left(A^{-1} F B^{-1} F^{\dagger}\right)^{k}\right] .
\end{aligned}
$$

The couplings $\{t\}$ and $\{\vec{t}\}$ are encoded in matrices $A, B$ by a Kontsevich-Miwa transformation

$$
t_{k}=\sum_{i=1}^{n} \frac{1}{k a_{i}^{k}}=\frac{1}{k} \operatorname{tr} A^{-k}, \quad \bar{t}_{k}=\sum_{j=1}^{n} \frac{1}{k b_{j}^{k}}=\frac{1}{k} \operatorname{tr} B^{-k} .
$$

To expand $Z(\{t\},\{\vec{t}\})$ in these variables, compute correlation functions with the even-valency vertices that appear in (5) for $k>1$ using the propagator from the matrix model

$$
\left\langle F_{j}^{i} F_{l}^{\dagger k}\right\rangle=\frac{\delta_{l}^{i} \delta_{j}^{k}}{\left(1-N a_{i}^{-1} b_{j}^{-1}\right)} .
$$

The color index for each face of the $F$ model Feynman diagrams comes with either an $a_{i}$ or a $b_{j}$, so the couplings

\footnotetext{
${ }^{2}$ See $[15,16]$ for reviews and references therein.
}

$\{t\}$ and $\{\vec{t}\}$ are associated to faces of the $F$ model. Thus, the $F$ model is of the $F$ type.

Relations between Hermitian matrix models via graph duality have appeared before in the literature, as have complex matrix models similar to the $F$ model (see for example [22-31], also in connection with $\tau$ functions [32,33]). The $F$ model is of the Kontsevich type because the couplings are encoded and expanded similarly to the Kontsevich model for topological gravity [3]. It is also of the Penner type because the appearance of a logarithmic term in the action is similar to the Penner model for the virtual Euler characteristic of the moduli space of punctured Riemann surfaces $\mathcal{M}_{g, n}$ [34]. In fact, the $F$ model is a complex matrix model analogue of the Hermitian Kontsevich-Penner model studied by Chekhov and Makeenko [35], which is dual to the Hermitian version of the $Z$ model (before the double-scaling limit) in exactly the same way [5,35].

The most direct way to prove the duality between the $Z$ and $F$ models is by using character expansions, see Sec. IV. Term by term it can be seen that the Feynman diagrams of the different models are graph dual. In Sec. IV D it is shown that the correlation functions are in fact sums over Hurwitz numbers, which count holomorphic maps from the world sheet to $\mathbb{C P} \mathbb{P}^{1}$ branched just 3 times. The valencies of the vertices and faces specify the ramification profiles.

Further proof is given in Sec. II with the techniques used in the 2D topological gravity case by Maldacena, Moore, Seiberg, Shih [10], and Gopakumar [5], which involve integrating fields in and out twice. In this method the graph duality between the $Z$ and $F$ model can be seen "dynamically," as explained in Sec. III. Every Feynman diagram in the original $Z$ matrix model corresponds to a diagram in the $F$ matrix model to which it is dual. This insight is crucial to read off the correct terms that are identified in the different models. In fact with the propagator (7), the $F$ model is only sensitive to "skeleton" graphs of the $Z$ model where propagators running parallel between the same vertices are bunched together into the same edge. These skeleton graphs were introduced in [36] as part of Gopakumar's program to find the closed string duals of free gauge theories [36-38].

An advantage of the $F$ model is that its correlation functions can be expressed directly as integrals over the moduli space $\mathcal{M}_{g, n}$ of punctured Riemann surfaces, using the example set by the Kontsevich model [3]. In the Schwinger parameterization of the propagators, the Schwinger lengths associated to each edge of each Feynman graph provide coordinates on a cell decomposition of $\mathcal{M}_{g, n}$. The integrals over the top-dimensional cells in $\mathcal{M}_{g, n}$ require all vertices of the graphs to be trivalent. The vertices of the $F$ model have a minimum valency of 4 , which means that the correlation functions can only come from lower-dimensional cells in the moduli space. Furthermore, following the analysis of the Hermitian 
Kontsevich-Penner model in [39], the integral localizes on discrete points in these subspaces, see Sec. V.

Despite the fact that the $Z$ model needs no doublescaling limit for its identification with the $c=1$ string, it is still possible to take one. From the $\mathcal{N}=4$ perspective, it is the Berenstein-Maldacena-Nastase (BMN) limit [40] and it limits the $F$ model to only 4-valent vertices, see Sec. IV C. The meaning of this limit for the $c=1$ string is unclear. Rewriting the correlation functions of the $Z / F$ model in terms of Hurwitz numbers in Sec. IV D, this limit involves restricting to a special class of Hurwitz numbers called double Hurwitz numbers, with arbitrary branching profiles at two points and simple branchings elsewhere.

Another topological matrix model for the $c=1, R=1$ string is the $W_{\infty}$ model of $[41,42]$, reviewed in [43], where just the positive momentum tachyon couplings are rearranged in this way

$$
Z(\{t\},\{\tilde{t}\})=\int[d M]_{N \times N}^{H} e^{\operatorname{tr}\left(-M+\sum_{k=1}^{\infty} \bar{t}_{k}\left(M A^{-1}\right)^{k}\right)} .
$$

The integral is over a Hermitian matrix $M$. The relation of the $Z$ model to this $W_{\infty}$ model was explained by Mukherjee and Mukhi in [44]; a direct transformation of the $W_{\infty}$ model into the $F$ model is shown in Appendix C.

\section{PROOF OF DUALITY USING INTEGRATION IN-OUT-IN-OUT}

In this section the duality between the $Z$ and $F$ models is proved using the techniques of $[5,10]$ by integrating in and out different fields. This makes the graph duality of the models manifest, as is explained in the next section.

The partition function for the $Z$ model is

$$
Z(\{t\},\{\vec{t}\})=\int[d Z]_{N \times N}^{\mathbb{C}} e^{-\operatorname{tr}\left(Z Z^{\dagger}\right)+\sum_{k=1}^{\infty} t_{k} \operatorname{tr}\left(Z^{k}\right)+\sum_{k=1}^{\infty} \bar{t}_{k} \operatorname{tr}\left(Z^{\dagger k}\right)} .
$$

This model is the same as the model II for the $c=1$ string at the self-dual radius $R=1$ with $N=\nu \equiv-i \mu$ in [14]. Although the integration in [14] is over a normal matrix with the condition $\left[Z, Z^{\dagger}\right]=0$ enforced, with this action for $R=1$ both the complex and normal matrix model are the same. ${ }^{3}$ The tachyon scattering matrix agrees with older results calculated in the literature [46].

Substitute the $t_{k}$ and $\bar{t}_{k}$ for two diagonal $n \times n$ matrices $A$ and $B$, with eigenvalues $a_{i}$ and $b_{j}$ respectively, using the Kontsevich-Miwa transformation

\footnotetext{
${ }^{3}$ A normal matrix can be decomposed into a unitary matrix $U$ and a diagonal matrix of its complex eigenvalues $D, Z_{N}=$ $U D U^{\dagger}$. This is not true for a complex matrix, for which we have $Z=U(D+R) U^{\dagger}$ where $R$ is strictly upper triangular [45]. It can be checked that in the action (9) $R$ completely decouples, and since the measure on $U$ and $D$ is the same, the normal matrix model is equivalent to the complex matrix model with this action. See Eq. (C4) in Appendix $\mathrm{C}$ for an alternative way to decompose a complex matrix.
}

$$
t_{k}=\frac{1}{k} \operatorname{tr} A^{-k}=\sum_{i=1}^{n} \frac{1}{k a_{i}^{k}}, \quad \bar{t}_{k}=\frac{1}{k} \operatorname{tr} B^{-k}=\sum_{j=1}^{n} \frac{1}{k b_{j}^{k}} .
$$

For the $t_{k}$ to be independent whenever the $\operatorname{tr}\left(Z^{k}\right)$ are, we need $n \geq N$ and similarly for the $\bar{t}_{k}$.

The exponentiated $\operatorname{tr}\left(Z^{k}\right)$ operators can be written as inverse determinants provided the $a_{i}$ are sufficiently large (to avoid convergence issues)

$$
\begin{aligned}
\exp \left[\sum_{k=1}^{\infty} t_{k} \operatorname{tr}\left(Z^{k}\right)\right] & =\exp \left[\sum_{k=1}^{\infty} \sum_{i=1}^{n} \frac{1}{k a_{i}^{k}} \operatorname{tr}\left(Z^{k}\right)\right] \\
& =\exp \left[-\sum_{i=1}^{n} \operatorname{tr} \log \left(1-\frac{Z}{a_{i}}\right)\right] \\
& =\prod_{i=1}^{n}\left[\operatorname{det}\left(1-\frac{Z}{a_{i}}\right)\right]^{-1} .
\end{aligned}
$$

[In the 2D topological gravity case the determinants in the double-scaled Hermitian matrix model correspond to exponentiated macroscopic loop operators for FateevZamolodchikov-Zamolodchikov-Teschner (FZZT) branes [47]

$$
\operatorname{tr} \log \left(a_{i}-M\right)=\int \frac{d l}{l} \operatorname{tr} e^{-l\left(a_{i}-M\right)} .
$$

Each of the $n$ FZZT branes has boundary cosmological constant $a_{i}$. There is no clear such interpretation of the determinants as wave functions of FZZT branes here, and in fact the more natural extension of [10] would be to investigate macroscopic loops in the MQM, cf. [48]. The fact that we have inverse determinants in the $c=1$ case (also present in the study of the normal matrix model in [44]) also differs from [10] and alters the statistics for the fields that we integrate in later, which in the $c<1$ case are fermionic strings stretching between the ZZ [49] and FZZT branes. ${ }^{4}$ From the $4 \mathrm{D} \mathcal{N}=4$ SYM perspective, these determinants [more clearly expanded in Eq. (48)] are interpreted as giant graviton branes in the bulk [19,50].]

Using (11), the $Z$ model partition function is now

$$
\begin{aligned}
Z(\{t\},\{\vec{t}\})= & \int[d Z]_{N \times N}^{\mathbb{C}} e^{-\operatorname{tr}\left(Z Z^{\dagger}\right)+\sum_{k=1}^{\infty} t_{k} \operatorname{tr}\left(Z^{k}\right)+\sum_{k=1}^{\infty} \bar{\tau}_{k} \operatorname{tr}\left(Z^{\dagger k}\right)} \\
= & \operatorname{det}(A)^{N} \operatorname{det}(B)^{N}\left\langle\prod_{i=1}^{n} \frac{1}{\operatorname{det}\left(a_{i}-Z\right)}\right. \\
& \left.\times \prod_{j=1}^{n} \frac{1}{\operatorname{det}\left(b_{j}-Z^{\dagger}\right)}\right\rangle
\end{aligned}
$$

\footnotetext{
${ }^{4}$ Note that if we had chosen to include a minus sign in the identification (10) we would have had normal determinants here and fermions integrated in later. The choice of sign is left to a physical interpretation in the future.
} 
The correlation function is taken with the Gaussian action $\operatorname{tr}\left(Z Z^{\dagger}\right)$, as will always be the case for the $Z$ model.

Writing the products of determinants using single determinants of larger $n N \times n N$ matrices, we can write them as integrals over two sets of complex bosonic fields ${ }^{5}$

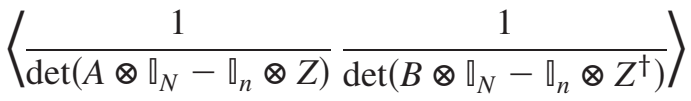

$$
\begin{aligned}
& =\int[d Z]_{N \times N}^{\mathbb{C}}[d C]_{N \times n}^{\mathbb{C}} \\
& \times[d D]_{N \times n}^{\mathbb{C}} e^{-\operatorname{tr}\left[Z Z^{\dagger}+C^{\dagger}\left(A \otimes \rrbracket_{N}-\mathbb{\square}_{n} \otimes Z\right) C+D^{\dagger}\left(B \otimes \rrbracket_{N}-\square_{n} \otimes Z^{\dagger}\right) D\right] .}
\end{aligned}
$$

$C_{e i}$ and $D_{e j}$ are bifundamental fields with $e=1, \ldots N$ and $i, j=1, \ldots n$. Again because we have inverse determinants this contrasts to the minimal string case, in which one must integrate in fermions rather than bosons.
Next integrate out the $Z$ field, after rewriting (14) appropriately

$$
\begin{aligned}
& \int[d Z][d C][d D] e^{-\operatorname{tr}\left[\left(Z-D D^{\dagger}\right)\left(Z^{\dagger}-C C^{\dagger}\right)-C C^{\dagger} D D^{\dagger}+C^{\dagger} A C+D^{\dagger} B D\right]} \\
& =\int[d C][d D] e^{-\operatorname{tr}\left(C^{\dagger} A C+D^{\dagger} B D-C C^{\dagger} D D^{\dagger}\right)} .
\end{aligned}
$$

This is the $C, D$ matrix model. The quartic vertex is $C C^{\dagger} D D^{\dagger}=C_{e i} C_{f i}^{\dagger} D_{f j} D_{e j}^{\dagger}$. It has propagators

$$
\left\langle C_{e i_{1}} C_{f i_{2}}^{\dagger}\right\rangle=\frac{\delta_{e f} \delta_{i_{1} i_{2}}}{a_{i_{1}}}, \quad\left\langle D_{e j_{1}} D_{f j_{2}}^{\dagger}\right\rangle=\frac{\delta_{e f} \delta_{j_{1} j_{2}}}{b_{j_{1}}} .
$$

The $C, D$ model can also be expanded as a function of the couplings $\{t\},\{\vec{t}\}$, see Appendix B.

Next we integrate back in an $n \times n$ complex matrix $F_{j}^{i}$ being careful with the indices

$$
\begin{aligned}
& \int[d C][d D] e^{-C_{e i_{1}}^{\dagger} A_{i_{1} i_{2}} C_{e i_{2}}-D_{e j_{1}}^{\dagger} B_{j_{1} j_{2}} D_{e j_{2}}+C_{e i} D_{e j}^{\dagger} D_{f j} C_{f i}^{\dagger}} \\
& =\int[d F]_{n \times n}^{\mathbb{C}}[d C][d D] e^{-\left(F_{j}^{i}-C_{e i} D_{e j}^{\dagger}\right)\left(F_{i}^{\dagger j}-D_{f j} C_{f i}^{\dagger}\right)+C_{e i} D_{e j}^{\dagger} D_{f j} C_{f i}^{\dagger}-C_{e i_{1}}^{\dagger} A_{i_{1} i_{2}} C_{e i_{2}}-D_{e j_{1}}^{\dagger} B_{j_{1} j_{2}} D_{e j_{2}}} \\
& =\int[d F]_{n \times n}^{\mathbb{C}}[d C][d D] e^{-\operatorname{tr}\left(F F^{\dagger}-D^{\dagger^{\dagger}} F^{\dagger} C-C^{\dagger} F D+C^{\dagger} A C+D^{\dagger} B D\right)} .
\end{aligned}
$$

To integrate out $C$ and $D$ write them together as a single $N \times 2 n$ field so that the cubic terms become

$$
\left(\begin{array}{cc}
C^{\dagger} & D^{\dagger}
\end{array}\right)\left(\begin{array}{cc}
A & -F \\
-F^{\dagger} & B
\end{array}\right)\left(\begin{array}{l}
C \\
D
\end{array}\right) .
$$

The result is an inverse determinant of an $[N \times 2 n] \times$ $[N \times 2 n]$ matrix

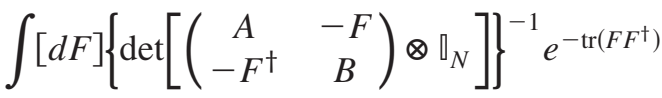

$$
\begin{aligned}
& =\int[d F]\left\{\operatorname{det}\left(\begin{array}{cc}
A & -F \\
-F^{\dagger} & B
\end{array}\right)\right\}^{-N} e^{-\operatorname{tr}\left(F F^{\dagger}\right)} .
\end{aligned}
$$

Now write the determinant in terms of a product of matrices

$$
\begin{aligned}
\operatorname{det}\left(\begin{array}{cc}
A & -F \\
-F^{\dagger} & B
\end{array}\right) & =\operatorname{det}\left(\left(\begin{array}{ll}
A & 0 \\
0 & B
\end{array}\right)\left(\begin{array}{cc}
1 & -A^{-1} F \\
-B^{-1} F^{\dagger} & 1
\end{array}\right)\right) \\
& =\operatorname{det}(A) \operatorname{det}(B) \operatorname{det}\left(\begin{array}{cc}
1 & -A^{-1} F \\
-B^{-1} F^{\dagger} & 1
\end{array}\right) .
\end{aligned}
$$

\footnotetext{
${ }^{5}$ This type of identity for the determinants only works with the determinant of a Hermitian matrix. We extend it to our case by noting that the $Z$ model only depends on the eigenvalues of $Z$ and since they are complex we can extend the identity by analytic continuation.
}

The constant terms $\operatorname{det}(A)^{-N} \operatorname{det}(B)^{-N}$ cancel those in (13), so we get

$$
Z(\{t\},\{\tilde{t}\})=\int[d F] e^{-\operatorname{tr}\left(F F^{\dagger}\right)-N \operatorname{tr} \log (}\left(\begin{array}{cc}
1 & -A^{-1} F \\
-B^{-1} F^{\dagger} & 1
\end{array}\right) .
$$

Expanding the logarithm for large $A, B$, just as we did in (11), we find

$$
\begin{aligned}
& Z(\{t\},\{\hat{t}\}) \\
& =\int[d F]_{n \times n}^{\mathbb{C}} e^{-\operatorname{tr}\left(F F^{\dagger}\right)+N \sum_{k=1}^{\infty}(1 / k) \operatorname{tr}}\left[\left(\begin{array}{cc}
0 & A^{-1} F \\
B^{-1} F^{\dagger} & 0
\end{array}\right)^{k}\right] \\
& =\int[d F]_{n \times n}^{\mathbb{C}} e^{-\operatorname{tr}\left(F F^{\dagger}\right)+N \sum_{k=1}^{\infty}(1 / k) \operatorname{tr}\left[\left(A^{-1} F B^{-1} F^{\dagger}\right)^{k}\right]} .
\end{aligned}
$$

This is the $F$ model introduced in Eq. (5). To extract the propagator study the quadratic term

$$
F_{j}^{i}\left(\delta_{i}^{l} \delta_{k}^{j}-N\left(A^{-1}\right)_{i}^{l}\left(B^{-1}\right)_{k}^{j}\right) F^{\dagger k}{ }_{l} .
$$

Using $\left(A^{-1}\right)_{i}^{l}=a_{i}^{-1} \delta_{i}^{l}$, the propagator is

$$
\left\langle F_{j}^{i} F_{l}^{\dagger k}\right\rangle=\frac{\delta_{l}^{i} \delta_{j}^{k}}{\left(1-N a_{i}^{-1} b_{j}^{-1}\right)} .
$$

Alternatively, we could have taken the plain quadratic term $\operatorname{tr}\left(F F^{\dagger}\right)$ with plain propagator 


$$
\left\langle F_{j}^{i} F_{l}^{\dagger k}\right\rangle_{\text {plain }}=\delta_{l}^{i} \delta_{j}^{k}
$$

and treated the $k=1$ term $N \operatorname{tr}\left(A^{-1} F B^{-1} F^{\dagger}\right)$ from (21) as an additional interaction vertex. The propagator (23) is then a sum over an arbitrary number of intervening such 2-valent vertices

$$
\begin{aligned}
& \sum_{p=0}^{\infty} \frac{N^{p}}{p !}\left\langle F_{j}^{i}\left[\operatorname{tr}\left(A^{-1} F B^{-1} F^{\dagger}\right)\right]^{p} F^{\dagger k}{ }_{l}\right\rangle_{\text {plain }} \\
& \quad=\sum_{p=0}^{\infty}\left(N a_{i}^{-1} b_{j}^{-1}\right)^{p} \delta_{l}^{i} \delta_{j}^{k}=\frac{\delta_{l}^{i} \delta_{j}^{k}}{\left(1-N a_{i}^{-1} b_{j}^{-1}\right)} .
\end{aligned}
$$

This is an important issue for the interpretation of the graph duality in Sec. III, since these 2-valent vertices are exactly those which are dual to the faces bounded by parallel propagators between the same vertices, see Fig. 7. Bunching such parallel propagators into a single edge, reducing the Feynman diagram to a skeleton graph, removes such 2-valent vertices from the dual graph. This works because the propagator (23) sums over all possible $Z$ diagrams with the same skeleton graph. These interpretational issues are also crucial to understand the dual of the planar two-point function of the $Z$ model and the character expansion to which we turn in Sec. IV.

It will often be useful for calculations to rescale the $F$ model $F \rightarrow \sqrt{A} F \sqrt{B}$ to get

$$
\begin{aligned}
Z(\{t\},\{\vec{t}\})= & (\operatorname{det} A)^{n}(\operatorname{det} B)^{n} \\
& \times \int[d F]_{n \times n}^{\mathbb{C}} e^{-\operatorname{tr}\left(A F B F^{\dagger}\right)+N} \sum_{k=1}^{\infty}(1 / k) \operatorname{tr}\left(F F^{\dagger}\right)^{k}
\end{aligned}
$$

This model then has the propagator

$$
\left\langle F_{j}^{i} F_{l}^{\dagger k}\right\rangle_{\text {scaled }}=\frac{\delta_{l}^{i} \delta_{j}^{k}}{a_{i} b_{j}-N} .
$$

This form is useful for transferring the Hermitian Kontsevich-Penner analysis of [39] to the $F$ model in Sec. V. Example $F$ model correlation functions are computed in Appendix A.

\section{DYNAMICAL GRAPH DUALITY}

In this section the duality between the $Z$ model and the $F$ model is shown to work at the level of individual Feynman diagrams. An explicit proof with formulae is provided in Sec. IV.

Each graph of the $Z$ model corresponds to a graph of the $F$ model which is dual to the original $Z$ model graph. The graph duality is shown "dynamically" in the sense that it is split up into stages where we first replace the vertices of the $Z$ model by faces (integrating in $C, D$ ), then contract and expand propagators in different channels (integrating out $Z$ and in $F$ ) and then finally replacing the faces of the $Z$ model by vertices of the $F$ model (integrating out $C, D$ ). This analysis completely mirrors the 2D topological gravity analysis by Gopakumar [5].

We start with a correlation function such as (3) for the $Z$ matrix model with several holomorphic vertices $\operatorname{tr}\left(Z^{k}\right)$ and several antiholomorphic vertices $\operatorname{tr}\left(Z^{\dagger k}\right)$. Each possible Wick contraction with the propagator will give a different Feynman graph. Because the only nontrivial propagator is $Z \rightarrow Z^{\dagger}$ and no vertices mix $Z$ 's with $Z^{\dagger}$ 's, there can be no propagators connecting a vertex back to itself. The Feynman diagram has a minimum genus surface on which it can be drawn with no lines crossing. Propagators that run parallel to each other between the same pair of vertices will be referred to as "homotopic" and can be bunched together into a single edge. If we do this for all possible propagators then we get what Gopakumar named a skeleton graph [36]. For each correlation function there may be several topologically distinct skeleton graphs, each corresponding to a number of possible Wick contractions of the original correlation function. Later in Sec. IV we will see that these distinct skeleton graphs correspond to the cut-and-join operators of [13].

It should also be noted that some graphs, where there is more than one single trace for both holomorphic and antiholomorphic operators, are disconnected; cf. the examples in Appendix A 4.

When we integrate in the $C, D$ matrices, a vertex like $\operatorname{tr}\left(Z^{m}\right)$ is replaced by a face of $C$ 's and an antiholomorphic vertex $\operatorname{tr}\left(Z^{\dagger m}\right)$ is replaced by a face of $D$ 's, see Fig. 1. In the figures the double lines of the $Z$ are drawn with solid lines
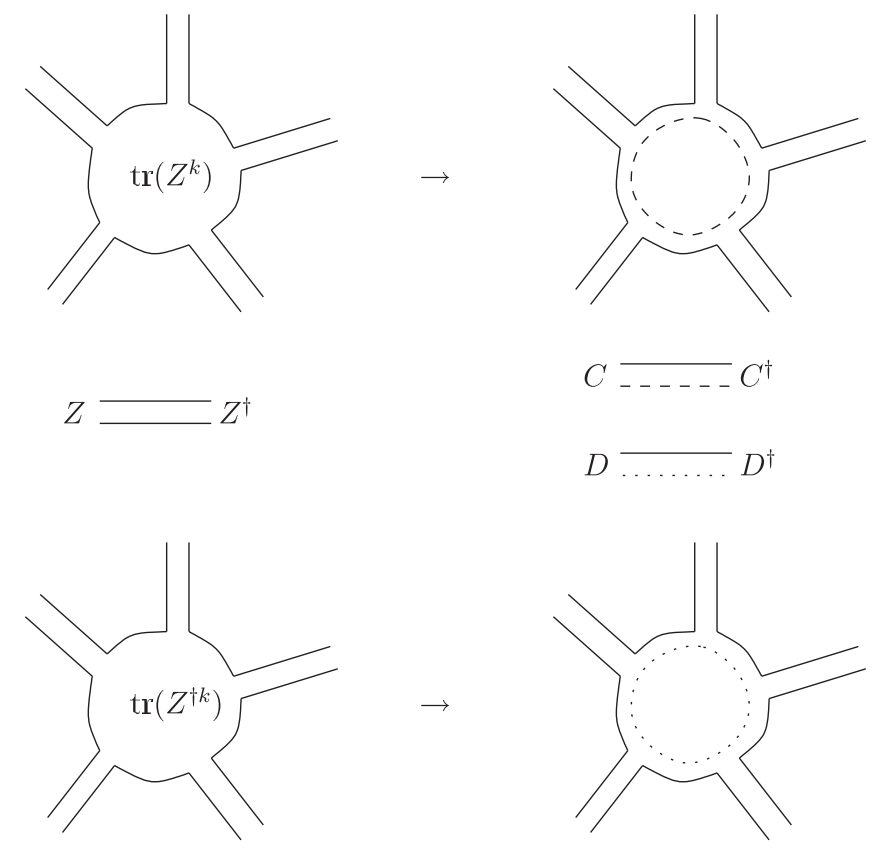

FIG. 1. $C, D$ integrated in. 


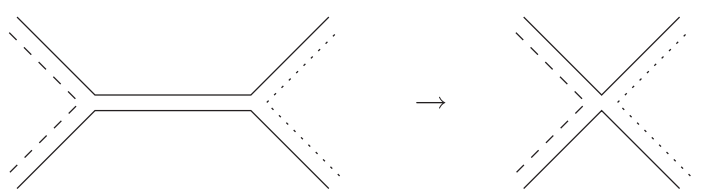

FIG. 2. $Z$ integrated out.
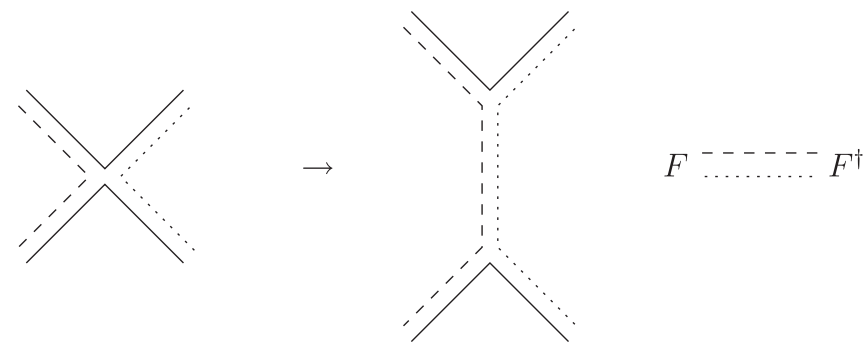

FIG. 3. $F$ integrated in.

$e=1, \ldots N$, while the bifundamental $C$ 's involve both a solid line and a dashed line for $i=1, \ldots n$ and the $D$ 's a solid line and a dotted line for $j=1, \ldots n$. We get cubic couplings $C^{\dagger} Z C$ and $D^{\dagger} Z^{\dagger} D$ from the action in (14).

We then integrate out $Z, Z^{\dagger}$. The $Z \rightarrow Z^{\dagger}$ propagators shrink to give graphs with the quartic vertex $C C^{\dagger} D D^{\dagger}$, cf. Figure 2. This is the $C, D$ matrix model from (15). It can be expanded in its own right, see the examples in Appendix B.

Integrating in the complex matrix $F$, the quartic vertex $C C^{\dagger} D D^{\dagger}$ expands in a different channel into an $F \rightarrow F^{\dagger}$ propagator with the cubic couplings $C^{\dagger} F D$ and $D^{\dagger} F^{\dagger} C$ of (17) at each end, cf. Fig. 3. In this way each propagator of the $Z$ model corresponds to a transverse propagator of the $F$ model via the quartic $C C^{\dagger} D D^{\dagger}$ vertex.

Finally we integrate out $C$ and $D$ to get the dual graph in terms of $F, F^{\dagger}$ for the $F$ model (21). Each face involving a solid line, corresponding to the original faces of the $Z$ model Feynman diagram, is replaced by an even-valency $F, F^{\dagger}$ vertex $\left(F F^{\dagger}\right)^{p}$, cf. Fig. 4 . Faces to which only two $F \rightarrow F^{\dagger}$ propagators connect become just a single $F \rightarrow F^{\dagger}$ propagator. These faces correspond to the faces between parallel homotopic propagators of the $Z$ model, cf. Fig. 7. Thus a bunch of parallel propagators from the $Z$ model become just a single $F$ propagator in the dual graph; in other words the $F$ model is sensitive only to the topology of the skeleton graph.

We have thus seen that the correspondence between the $Z$ model and the $F$ model corresponds to graph duality. A vertex of the $Z$ model becomes a face of the $F$ model; homotopically bunched propagators (edges) of the $Z$ model become a single propagator (edge) of the $F$ model which is perpendicular to the original $Z \rightarrow Z^{\dagger}$ propagators; and finally the faces of the $Z$ model become even-valency vertices of the $F$ model.
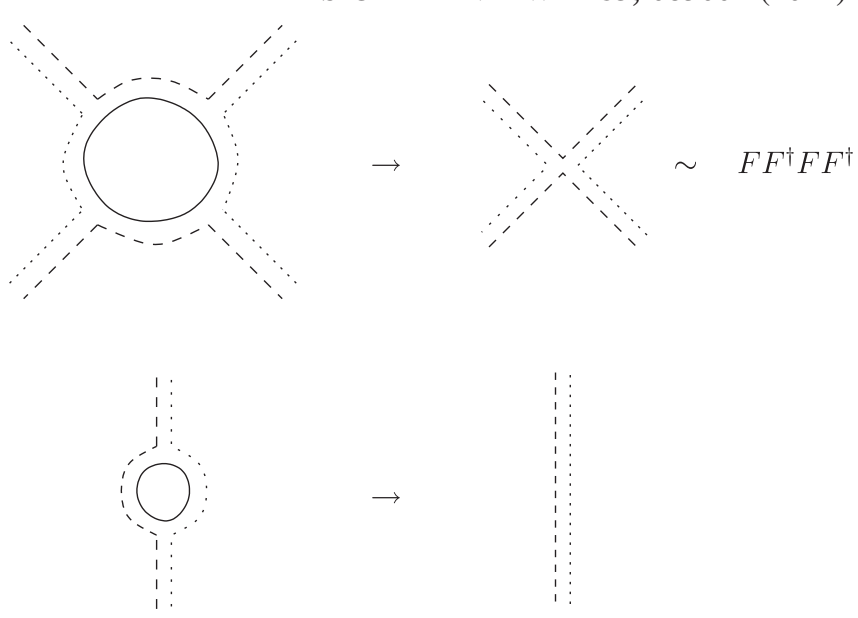

FIG. 4. $C, D$ integrated out; note that you can have any evenvalency $\left(F F^{\dagger}\right)^{p}$ of $F, F^{\dagger}$ vertices.

An important constraining feature of the $F$ model (21) is that the faces of the graphs are always associated with either A's or $B$ 's but never both. From the propagator in (23) the $a_{i}$ is associated to one (dashed) index line, while the $b_{j}$ is associated to the other (dotted) index line, cf. the left part of Fig. 5. The vertices also preserve the $a_{i}$ and $b_{j}$ associations, cf. the right part of Fig. 5. This is because the faces correspond to vertices of the $Z$ model where the $A$ 's map to $\operatorname{tr}\left(Z^{k}\right)$ vertices and the $B$ 's to $\operatorname{tr}\left(Z^{\dagger k}\right)$ vertices. In fact, because a $Z$ model vertex can never have a selfcontraction, each edge of the $F$ model has an $A$ face on one side and a $B$ face on the other, reflected in the fact that the double-line propagator for $F$ has one dashed and one dotted line.

A full example of this dynamical graph duality is given for $\left\langle\operatorname{tr}\left(Z^{2}\right) \operatorname{tr}(Z) \operatorname{tr}\left(Z^{\dagger 3}\right)\right\rangle$ in Fig. 6 .

To conclude this section we summarize the procedure for seeing the duality from graphs:

(i) Starting from the $Z$ model, expand the partition function in correlation functions of holomorphic and antiholomorphic operators. Each correlation function corresponds to a sum of different topologically
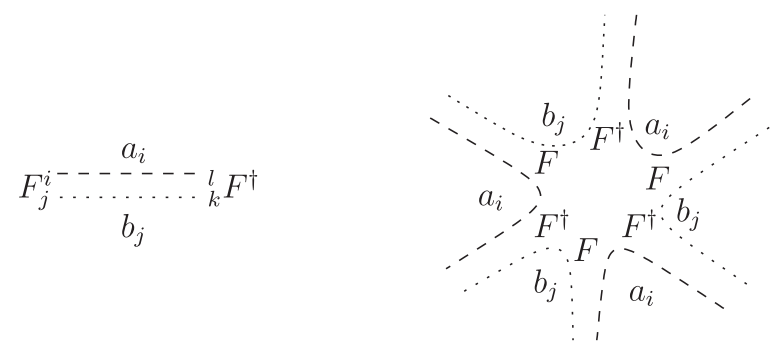

FIG. 5. Associations of $A$ with dashed faces and $B$ with dotted faces. On the left is the propagator and associated $a_{i}, b_{j}$. On the right is an example 6-valent vertex $\operatorname{tr}\left[\left(A^{-1} F B^{-1} F^{\dagger}\right)^{3}\right]$, also with associated $a_{i}, b_{j}$. 


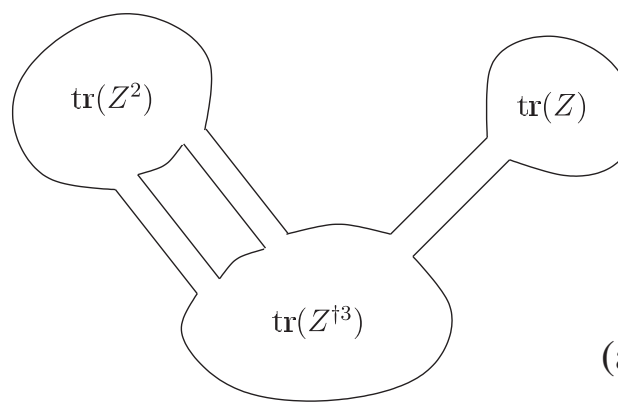

(a)
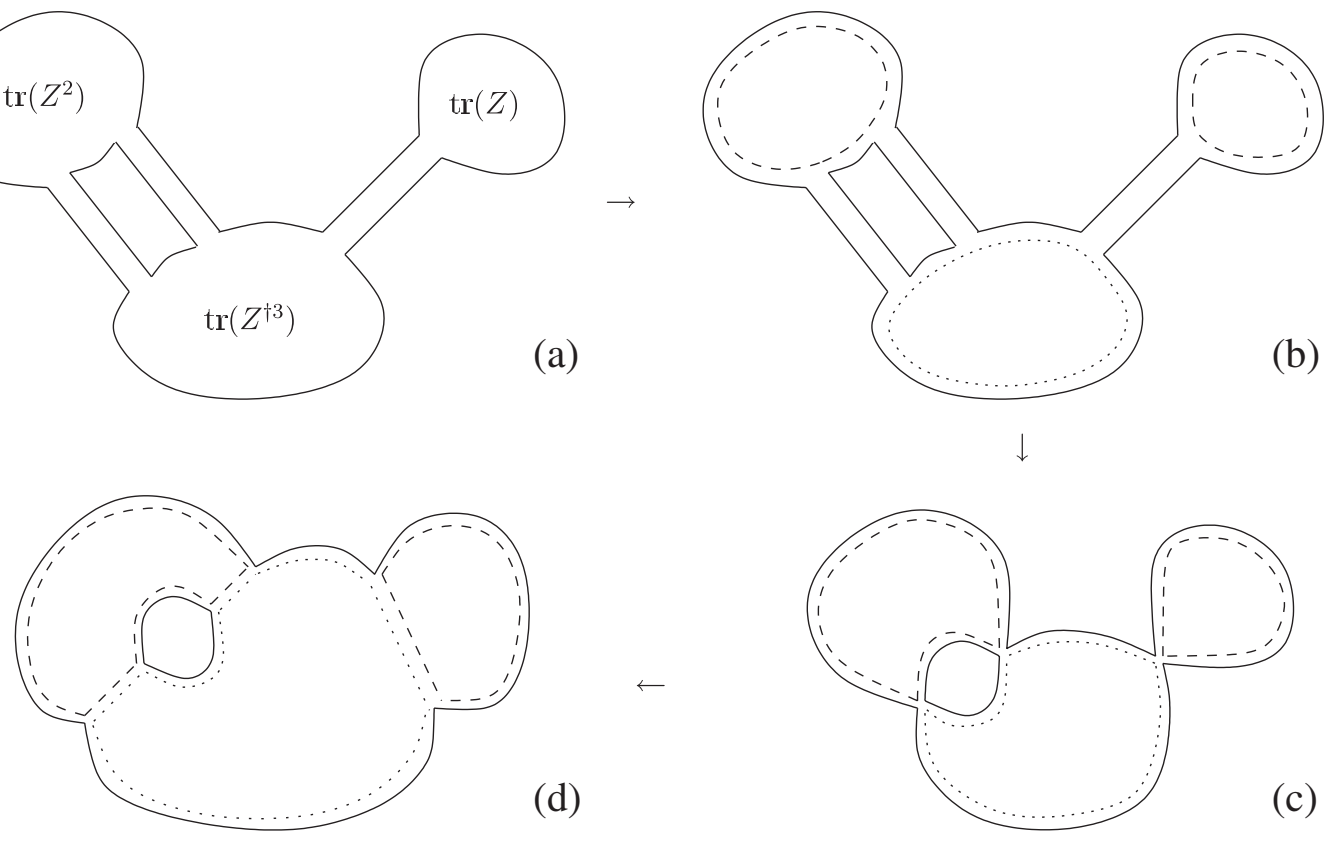

(d)
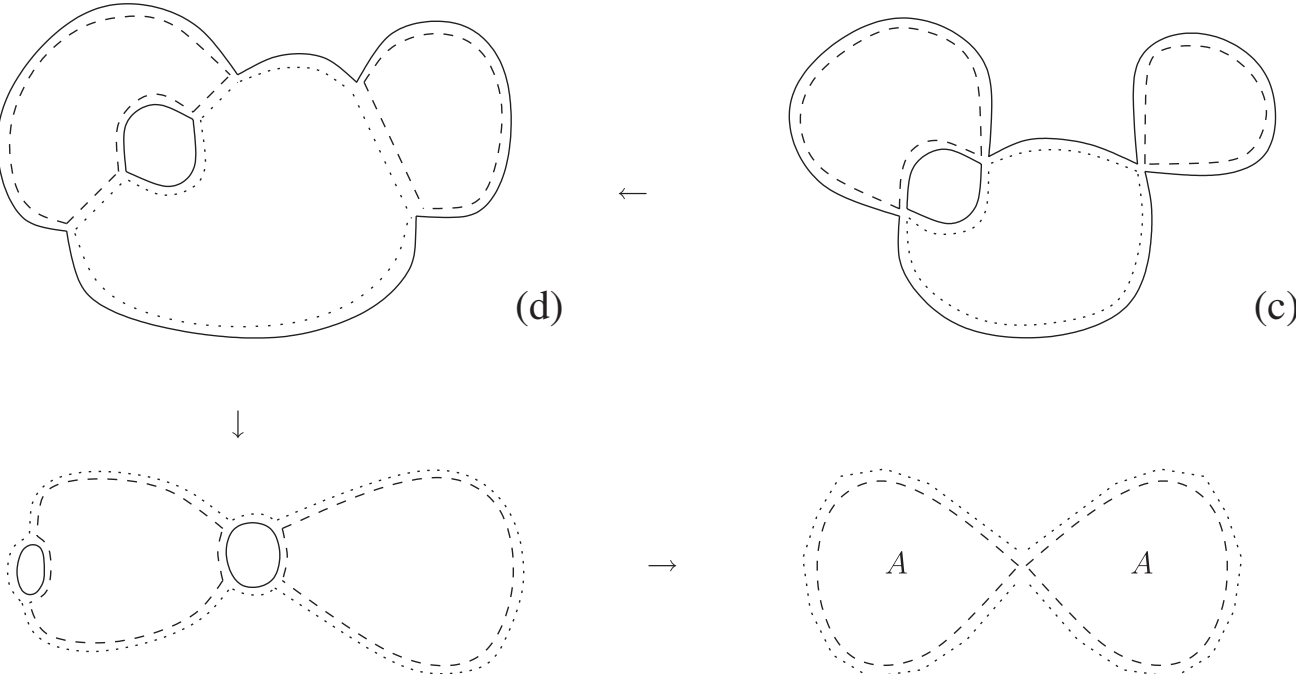

(e)

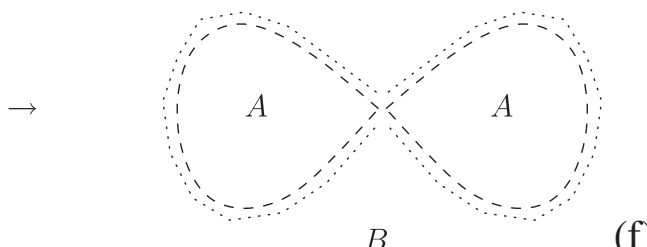

(f)

FIG. 6. $\left\langle\operatorname{tr}\left(Z^{2}\right) \operatorname{tr}(Z) \operatorname{tr}\left(Z^{\dagger 3}\right)\right\rangle$ graph duality, step by step. Graph (a) is the original $Z$ model double-line diagram with three vertices and three propagators. In (b) $C$ and $D$ are integrated in, replacing the vertices of $Z$ with faces of $C$ and $D$. In (b) the $Z$ propagators are shrunk to give the quartic vertices $C C^{\dagger} D D^{\dagger}$ of the $C, D$ model. In (d) the quartic vertices are expanded in a different channel as propagators of $F$. Figure part (e) is the same graph as (d), just redrawn on the sphere so that the outer solid line of (d) becomes the inside central solid line of (e). In (f) $C$ and $D$ are integrated out; the faces of solid lines in (e) have been replaced by $F / F^{\dagger}$ vertices. Now faces of (f) bounded by dashed lines are associated with $A$ 's [corresponding to holomorphic vertices of (a)], while faces bounded by dotted lines are associated with $B$ 's [corresponding to the antiholomorphic vertices of (a)].

distinct skeleton graphs. Each skeleton graph corresponds to a sum of topologically identical $F$ model Feynman diagrams, to which the skeleton graph is dual.

(ii) Starting from the $F$ model, expand the partition function in correlation functions of the interaction vertices. Each $F$ correlation function splits into classes of topologically identical Feynman diagrams. Each such class of Feynman diagrams is dual to a family of topologically identical $Z$ skeleton graphs.

We will now give an alternative proof of this graph duality using symmetric group techniques.

\section{CHARACTER EXPANSIONS}

In this section we use symmetric group and representation theory techniques to expand both the $Z$ and $F$ models and show that they are equal. The graph duality is revealed in the character expansions of the models. In fact a more general correspondence is proven

$$
\begin{aligned}
& Z(\{t\},\{\bar{t}\},\{s\}) \\
& =\int[d Z]_{N \times N}^{\mathbb{C} \times N} \\
& \quad \times e^{-\operatorname{tr}\left(S^{-(1 / 2)} Z S^{-(1 / 2)} Z^{\dagger}\right)+\sum_{k=1}^{\infty} t_{k} \operatorname{tr}\left(Z^{k}\right)+\sum_{k=1}^{\infty} \bar{t}_{k} \operatorname{tr}\left(Z^{\dagger k}\right)}, \\
& =\sum_{l(R) \leq N} \frac{|R| ! \chi_{R}(S) \chi_{R}\left(A^{-1}\right) \chi_{R}\left(B^{-1}\right)}{d_{R}}
\end{aligned}
$$

$$
=\int[d F]_{n \times n}^{\mathbb{C}} e^{-\operatorname{tr} F F^{\dagger}+\sum_{k=1}^{\infty} s_{k} \operatorname{tr}\left(A^{-1} F B^{-1} F^{\dagger}\right)^{k}},
$$




$$
\propto \int[d F]_{n \times n}^{\mathbb{C}} e^{-\operatorname{tr}\left(A F B F^{\dagger}\right)+\sum_{k=1}^{\infty} s_{k} \operatorname{tr}\left(F F^{\dagger}\right)^{k}} .
$$

In the first line the quadratic term of the $Z$ model has been modified with an $N \times N$ diagonal matrix $S$ whose eigenvalues are $S_{1} \cdots S_{N}$. The propagator is now

$$
\left\langle Z_{f}^{e} Z^{\dagger g}{ }_{h}\right\rangle=S_{e}^{1 / 2} S_{f}^{1 / 2} \delta_{h}^{e} \delta_{f}^{g} .
$$

Each face of the $Z$ model is bounded by $2 k$ edges (the face has valency $2 k$ ). Since each edge picks up a factor of $S^{1 / 2}$ from the propagator, each $2 k$-valent face of the $Z$ model comes with a factor $\operatorname{tr}\left(S^{k}\right)$. Each vertex of the $Z$ model has a coupling $t_{k}$ or $\bar{t}_{k}$ depending on whether it is holomorphic or antiholomorphic. To recover the vanilla $Z$ model of (1) set $S$ to be the identity matrix $S=\rrbracket_{N}$.

In the second line the $Z$ model has been expanded in characters $\chi_{R}$ of the group $U(N)$ whose representations $R$ are labeled by Young diagrams with at most $N$ rows, $l(R) \leq N .|R|$ is the number of boxes in the Young diagram and $d_{R}$ is the dimension of the symmetric group representation for the same Young diagram. If we set $S=\rrbracket_{N}$ here, the character of $S$ becomes the dimension of the $U(N)$ representation $R, \chi_{R}\left(\square_{N}\right)=\operatorname{dim}_{N} R$. Note the symmetry of the character expansion in the variables $\{t\},\{\vec{t}\}$ and $\{s\}$, as encoded in the matrices $A^{-1}, B^{-1}$ and $S$.

In the third line this character expansion is equal to the $F$ model, which has been modified so that now each $2 k$-valent vertex is weighted with a coupling $s_{k}$. This coupling is related to the $N \times N$ matrix $S$ by the Kontsevich-Miwa transformation $s_{k} \equiv \frac{1}{k} \operatorname{tr}\left(S^{k}\right)$. If we set $S=\rrbracket_{N}$ here, $s_{k}$ becomes $s_{k}=\frac{1}{k} \operatorname{tr}\left(\unrhd_{N}^{k}\right)=\frac{N}{k}$ and we recover the vanilla $F$ model of (5).

In the fourth line the $F$ model has been rescaled $F \rightarrow$ $\sqrt{A} F \sqrt{B}$ as in Eq. (26) to make the duality with the $Z$ model in (28) more transparent.

In the $Z$ model each $2 k$-valent face picks up a coupling $s_{k}$ from the propagator, while the $k$-valent vertices pick up couplings $t_{k}$ or $\bar{t}_{k}$. On the other hand, for the dual $F$ model, exactly the reverse happens: it is the $2 k$-valent vertices which pick up the couplings $s_{k}$, while the $k$-valent faces pick up couplings $t_{k}$ or $\bar{t}_{k}$ from the propagator. Thus, the addition of the matrix $S$ and its associated couplings $s_{k}$ allow us to follow the graph duality more clearly.

Similar Hermitian models where faces of their graph expansions are weighted separately from the vertices have been studied in [23-26] and indeed the $F$ model and its character expansion were mentioned in the review [27] as a complex generalization.

\section{A. Character expansion of the $\boldsymbol{Z}$ model}

In this section the symmetric group techniques of $[13,19]$ are used to expand the $Z$ model, which generates the correlation functions computed in those papers.

The first step is to write each product of holomorphic traces (often referred to as multitrace operators) using a conjugacy class element of $S_{k}$ where $k$ is the sum of the powers of $Z$. We write the conjugacy class as a partition $\left[\mu_{1}, \mu_{2}, \cdots \mu_{p}\right]$ of $k$ so that $k=\sum_{i} \mu_{i} \equiv|\mu|{ }^{6}$ Each $\mu_{i}$ corresponds to a $\mu_{i}$ cycle in $S_{k}$. In this way

$$
\begin{aligned}
\operatorname{tr}\left(Z^{\mu_{1}}\right) \operatorname{tr}\left(Z^{\mu_{2}}\right) \cdots \operatorname{tr}\left(Z^{\mu_{p}}\right) & =Z_{e_{\alpha(1)}}^{e_{1}} Z_{e_{\alpha(2)}}^{e_{2}} \cdots Z_{e_{\alpha(k)}}^{e_{k}} \\
& \equiv \operatorname{tr}\left(\alpha Z^{\otimes k}\right),
\end{aligned}
$$

where $\alpha$ is in the conjugacy class $\left[\mu_{1}, \mu_{2}, \cdots \mu_{p}\right]$. For a partition $\mu$ of $k$ (written $\mu \vdash k$ ), we write a representative of the corresponding conjugacy class $[\mu]$ as $\alpha_{\mu} \in[\mu] \subset$ $S_{k}$. In fact for these operators it does not matter which representative we pick because all elements in the conjugacy class $[\mu]$, given by $\rho^{-1} \alpha_{\mu} \rho$ for $\rho \in S_{k}$, correspond to the same multitrace operator (33). The size of the conjugacy class is

$$
|[\mu]|=\frac{k !}{|\operatorname{Sym}([\mu])|}=\frac{k !}{\prod_{p=1}^{k} p^{i_{p}(\mu)} i_{p}(\mu) !} .
$$

$|\operatorname{Sym}([\mu])|$ is the size of the symmetry group $\operatorname{Sym}([\mu])$ of the conjugacy class. $\operatorname{Sym}\left(\alpha_{\mu}\right)$ is the subgroup of $S_{k}$ that leaves $\alpha_{\mu}$ invariant under conjugation: $\operatorname{Sym}\left(\alpha_{\mu}\right)=\{\rho \in$ $\left.S_{k} \mid \rho \alpha_{\mu} \rho^{-1}=\alpha_{\mu}\right\} . i_{p}(\mu)$ is the number of parts of $\mu$ of length $p$. The factors in the denominator of (34) come from the cyclic symmetry $\cong \mathbb{Z}_{p}$ of the $i_{p}(\mu)$ cycles of length $p$ and the $i_{p}(\mu)$ ! ways of exchanging them.

Summing over all permutations of the Wick contractions of the fields, the correlation functions of the vanilla $Z$ model (1) are an expansion in $N$ where the power of $N$ is the number of faces of the $Z$ model graph [19]

$$
\begin{aligned}
\left\langle\operatorname{tr}\left(\alpha_{\nu} Z^{\dagger \otimes k}\right) \operatorname{tr}\left(\alpha_{\mu} Z^{\otimes k}\right)\right\rangle & =\sum_{\tau \in S_{k}} N^{C\left(\alpha_{\mu} \tau \alpha_{\nu} \tau^{-1}\right)} \\
& =\sum_{\sigma, \tau \in S_{k}} N^{C(\sigma)} \delta\left(\sigma \alpha_{\mu} \tau \alpha_{\nu} \tau^{-1}\right) .
\end{aligned}
$$

$C(\sigma)$ is the number of cycles in the permutation $\sigma \in S_{k}$ (i.e. the number of parts of the corresponding partition). The second equality is just a rewriting of the first with the symmetric group delta function $\delta(\sigma) . \delta(\sigma)$ is zero on all elements of $S_{k}$ except the group identity id, on which it is 1 .

Since the operators only depend on the conjugacy of $\alpha_{\mu}$, it is often useful to write them with sums over the entire conjugacy class $\operatorname{tr}\left(\alpha_{\mu} Z^{\otimes k}\right)=\frac{1}{[[\mu] \mid} \operatorname{tr}\left(\Sigma_{\mu} Z^{\otimes k}\right)$, where

$$
\Sigma_{\mu} \equiv \sum_{\alpha \in[\mu] \subset S_{k}} \alpha .
$$

The sum over $\sigma \in S_{k}$ in (35) can also be subdivided into conjugacy classes

\footnotetext{
${ }^{6}$ Assume the parts of the partition are ordered $\mu_{i} \geq \mu_{i+1}$ so that the parts map to the row lengths of a Young diagram with $k$ boxes.
} 

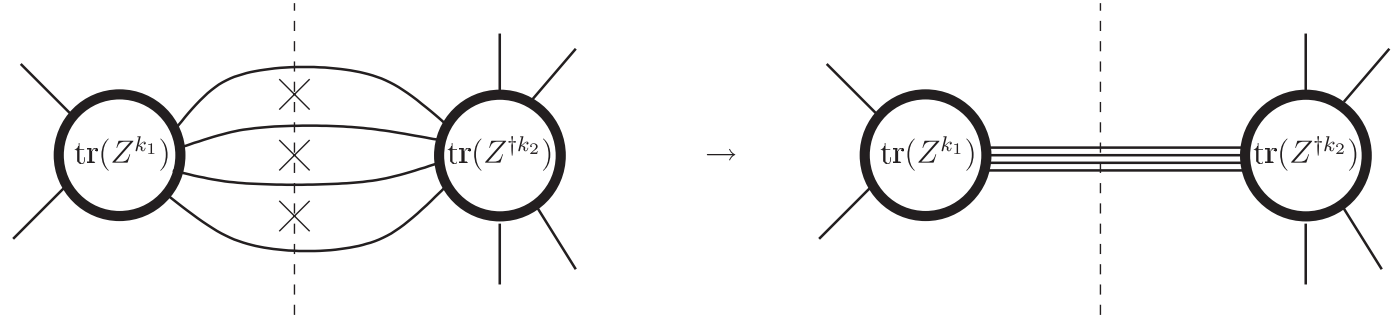

FIG. 7. Replacement of 2-valent vertices of the $F$ model with plain $\operatorname{tr}\left(F F^{\dagger}\right)$ quadratic term (dashed lines), dual to faces bounded by parallel propagators in the $Z$ model (thin solid lines), by the propagator of the proper $F$ model (23), which is dual to the parallel propagators of the $Z$ model bunched into an edge of the skeleton graph. [All propagators are drawn in a single-line notation here for ease of reading.]

$$
\sum_{\sigma \in S_{k}} N^{C(\sigma)} \sigma=\sum_{\lambda \vdash k} N^{C([\lambda])} \Sigma_{\lambda}
$$

This element of the group algebra is often written in the literature as

$$
\Omega_{k} \equiv N^{-k} \sum_{\lambda \vdash k} N^{C([\lambda])} \Sigma_{\lambda}=\sum_{\lambda \vdash k} \frac{1}{N^{T([\lambda])}} \Sigma_{\lambda} .
$$

We have used the identity $T([\lambda])=k-C([\lambda])$, where $T([\lambda])$ is the minimum number of transpositions needed to build an element in the conjugacy class $[\lambda]$.

The correlation function (35) can now be written as

$$
\begin{aligned}
\left\langle\operatorname{tr}\left(\alpha_{\nu} Z^{+\otimes k}\right) \operatorname{tr}\left(\alpha_{\mu} Z^{\otimes k}\right)\right\rangle & =\frac{k ! N^{k}}{|[\mu]||[\nu]|} \delta\left(\Sigma_{\nu} \Omega_{k} \Sigma_{\mu}\right) \\
& =\frac{k !}{|[\mu]||[\nu]|} \sum_{\lambda \vdash k} N^{C([\lambda])} \delta\left(\Sigma_{\nu} \Sigma_{\lambda} \Sigma_{\mu}\right) .
\end{aligned}
$$

Each summand in (39) now corresponds to topologically different graphs. The partitions $\mu, \nu$ label the different holomorphic and antiholomorphic vertices, respectively. The partition $\lambda$, on the other hand, labels the faces. Each part $\lambda_{i}$ of the partition labels a face so that $C([\lambda])$ is the total number of faces and hence gives the power of $N$. Putting a nontrivial matrix $S$ into the $Z$ model as in (28) the factor $N^{C([\lambda])}$ is refined as

$$
N^{C([\lambda])} \rightarrow \operatorname{tr}\left(\alpha_{\lambda} S^{\otimes k}\right)=\prod_{p=1}^{k}\left[\operatorname{tr}\left(S^{p}\right)\right]^{i_{p}(\lambda)} .
$$

$i_{p}(\lambda)$ is the number of parts in $\lambda$ of length $p$. Each part of length $p$ corresponds to a $2 p$-valent face of the $Z$ graph bounded by $2 p$ edges. The parts of length 1 correspond to faces with only two edges; these are the faces between propagators running parallel between two vertices, see the left-hand diagram in Fig. 7. Bunching these parallel propagators into single edges so that we get a skeleton graph that corresponds to ignoring the parts of $\lambda$ of length 1 . This proves a conjecture in [13] that the different $\Sigma_{\lambda}$ correspond to the different skeleton graphs if you ignore the parts of length 1 . The genus of the Feynman diagram can be read off using the Euler characteristic with $V=C([\mu])+$ $C([\nu])$ vertices, $E=k$ edges and $F=C([\lambda])$ faces

$$
\begin{aligned}
\chi & =2-2 g=V-E+F \\
& =C([\mu])+C([\nu])+C([\lambda])-k .
\end{aligned}
$$

In [13] the $\Sigma_{\lambda}$ were called cut-and-join operators because they have an action on a multitrace operator $\operatorname{tr}\left(\Sigma_{\mu} Z^{\otimes k}\right)$ that can split single traces into many traces or join many into one. This action is simply left multiplication

$$
\Sigma_{\lambda}: \operatorname{tr}\left(\Sigma_{\mu} Z^{\otimes k}\right) \rightarrow \operatorname{tr}\left(\Sigma_{\lambda} \Sigma_{\mu} Z^{\otimes k}\right) .
$$

For example, the conjugacy class of transpositions $\lambda=$ $\left[2,1^{k-2}\right]$ gives the cut-and-join operator $\Sigma_{[2]}$. Acting on the single-trace operator given by $\mu=[k]$ splits it into all possible double traces $[p, k-p]$

$\Sigma_{[2]} \operatorname{tr}\left(Z^{k}\right)=\frac{1}{|[k]|} \operatorname{tr}\left(\Sigma_{[2]} \Sigma_{[k]} Z^{\otimes k}\right)=\frac{k}{2} \sum_{p=1}^{k-1} \operatorname{tr}\left(Z^{p}\right) \operatorname{tr}\left(Z^{k-p}\right)$.

Acting on a double trace with $\Sigma_{[2]}$ can also join it into a single trace. Similar results follow when acting with more general cut-and-join operators $\Sigma_{\lambda}$ on more general trace structures. When writing partitions $\lambda$ for cut-and-join operators we omit parts of length 1 so that in this example $\lambda=\left[2,1^{k-2}\right] \rightarrow[2]$. This omission of length-1 parts corresponds to only considering skeleton graphs, as discussed above. $^{7}$

Next we turn to the character expansion. The symmetric group delta function in (39) can be expanded as a sum over representations $R$ of $S_{k}$, also indexed by partitions, in terms of the characters $\chi_{R}(\sigma)$ of $\sigma$ in the representations $R$ and the $S_{k}$ representation dimensions $d_{R}=\chi_{R}(\mathrm{id})$. The identity is $\delta(\sigma)=\frac{1}{k !} \sum_{R \vdash k} d_{R} \chi_{R}(\sigma)$. Using the fact that

\footnotetext{
${ }^{7}$ In [13] it was useful to think of the correlation function (39) as an inner product of $\operatorname{tr}\left(\alpha_{\nu} Z^{\dagger \otimes k}\right)$ with the result of $\Omega_{k}$ acting on $\operatorname{tr}\left(\alpha_{\mu} Z^{\otimes k}\right)$. The bra-ket notation of the inner product in [13] is the same as the delta function here $\langle\nu \mid \mu\rangle=\frac{k !}{\| \mu] \| \nu]} \delta\left(\Sigma_{\mu} \Sigma_{\nu}\right)=$ $|\operatorname{Sym}([\mu])| \delta_{\mu=\nu}$.
} 
$d_{R} \chi_{R}\left(\Sigma_{\mu} \sigma\right)=\chi_{R}(\sigma) \chi_{R}\left(\Sigma_{\mu}\right)$, since $\Sigma_{\mu}$ is central in $S_{k}$, and the relation between $\Omega_{k}$ and the unitary group $U(N)$ dimension of the partition $R, N^{k} \chi_{R}\left(\Omega_{k}\right)=k ! \operatorname{dim}_{N} R,{ }^{8}$ the correlation function (39) has a character expansion

$$
\left\langle\operatorname{tr}\left(\alpha_{\nu} Z^{\dagger \otimes k}\right) \operatorname{tr}\left(\alpha_{\mu} Z^{\otimes k}\right)\right\rangle=\sum_{R \vdash k} \frac{k ! \chi_{R}\left(\alpha_{\mu}\right) \chi_{R}\left(\alpha_{\nu}\right) \operatorname{dim}_{N} R}{d_{R}} .
$$

This identity can also be seen by expanding the traces in terms of $U(N)$ characters $\operatorname{tr}\left(\alpha_{\mu} Z^{\otimes k}\right)=\sum_{R \vdash k} \times$ $\chi_{R}\left(\alpha_{\mu}\right) \chi_{R}(Z)$, where the $U(N)$ character $\chi_{R}(Z)$ is itself expanded in terms of traces $\chi_{R}(Z)=\frac{1}{k !} \sum_{\alpha \in S_{k}} \times$ $\chi_{R}(\alpha) \operatorname{tr}\left(\alpha Z^{\otimes k}\right)$. The two-point function of these characters $\chi_{R}(Z)$ is diagonal [19]. Note that the $\operatorname{dimension}^{\operatorname{dim}_{N} R}$ vanishes if the number of parts $l(R)$ of the partition $R$ exceeds $N$.

For general $S$ as in (28) the correlation functions (39) and (44) become

$$
\begin{aligned}
\left\langle\operatorname{tr}\left(\alpha_{\nu} Z^{\dagger \otimes k}\right) \operatorname{tr}\left(\alpha_{\mu} Z^{\otimes k}\right)\right\rangle= & \sum_{R \vdash k} \sum_{\lambda \vdash k}|[\lambda]| \operatorname{tr}\left(\alpha_{\lambda} S^{\otimes k}\right) \\
& \times \frac{\chi_{R}\left(\alpha_{\mu}\right) \chi_{R}\left(\alpha_{\nu}\right) \chi_{R}\left(\alpha_{\lambda}\right)}{d_{R}}, \\
= & \sum_{R \vdash k} \frac{k ! \chi_{R}\left(\alpha_{\mu}\right) \chi_{R}\left(\alpha_{\nu}\right) \chi_{R}(S)}{d_{R}} .
\end{aligned}
$$

Now we are in a position to expand the full $Z$ partition function. It is useful to collect the expansions of the exponentials into multitrace operators indexed by partitions $\mu$ of $k$, so that the total number of $Z$ fields in each term is $k$. Using the Kontsevich-Miwa transformation (10)

$$
\begin{aligned}
e^{\sum_{k=1}^{\infty} t_{k} \operatorname{tr}\left(Z^{k}\right)} & =\sum_{k=0}^{\infty} \sum_{\mu \vdash k} \prod_{p=1}^{k} \frac{\left[t_{p} \operatorname{tr}\left(Z^{p}\right)\right]^{i_{p}(\mu)}}{i_{p}(\mu) !} \\
& =\sum_{k=0}^{\infty} \sum_{\mu \vdash k} \prod_{p=1}^{k} \frac{\left[\operatorname{tr}\left(A^{-p}\right) \operatorname{tr}\left(Z^{p}\right)\right]^{i_{p}(\mu)}}{k^{i_{p}(\mu)} i_{p}(\mu) !} .
\end{aligned}
$$

The denominator factors give the size of the conjugacy class from (34) so

$$
\begin{aligned}
e^{\sum_{k=1}^{\infty} t_{k} \operatorname{tr}\left(Z^{k}\right)} & =\sum_{k=0}^{\infty} \sum_{\mu \vdash k} \frac{|[\mu]|}{k !} \operatorname{tr}\left(\alpha_{\mu}\left(A^{-1}\right)^{\otimes k}\right) \operatorname{tr}\left(\alpha_{\mu} Z^{\otimes k}\right) \\
& =\sum_{k=0}^{\infty} \sum_{R \vdash k} \chi_{R}\left(A^{-1}\right) \chi_{R}(Z) .
\end{aligned}
$$

The $Z$ model partition function (28) becomes

\footnotetext{
${ }^{8}$ Characters of the cut-and-join operators $\chi_{R}\left(\Sigma_{\mu}\right)$ roughly correspond to $U(N)$ Casimirs, cf. Sec. 2.7 of [13] and the original references in $[51,52]$.
}

$$
\begin{aligned}
Z(\{t\},\{\bar{t}\})= & \left\langle e^{\sum_{k=1}^{\infty} \bar{t}_{k} \operatorname{tr}\left(Z^{\dagger k}\right)} e^{\sum_{k=1}^{\infty} t_{k} \operatorname{tr}\left(Z^{k}\right)}\right\rangle \\
= & \sum_{k=0}^{\infty} \sum_{\mu, \nu \vdash k} \frac{|[\mu]|}{k !} \operatorname{tr}\left(\alpha_{\mu}\left(A^{-1}\right)^{\otimes k}\right) \\
& \times \frac{|[\nu]|}{k !} \operatorname{tr}\left(\alpha_{\nu}\left(B^{-1}\right)^{\otimes k}\right) \\
& \times\left\langle\operatorname{tr}\left(\alpha_{\nu} Z^{\dagger \otimes k}\right) \operatorname{tr}\left(\alpha_{\mu} Z^{\otimes k}\right)\right\rangle \\
= & \sum_{k=0}^{\infty} \sum_{R \vdash k} \frac{k ! \chi_{R}\left(A^{-1}\right) \chi_{R}\left(B^{-1}\right) \chi_{R}(S)}{d_{R}} .
\end{aligned}
$$

Noting that $\chi_{R}(S)$ for an $N \times N$ matrix $S$ vanishes if $l(R)>N$, and since $n \geq N$ this constraint takes precedence over those coming from $\chi_{R}\left(A^{-1}\right)$ and $\chi_{R}\left(B^{-1}\right)$, this concludes the proof of (29).

\section{B. Character expansion of the $\boldsymbol{F}$ model}

A generalization of the Itzykson-Zuber integral [53] (see for example [31]) can be used for the following integral for the $F$ model with plain quadratic term (24):

$$
\begin{aligned}
& \left\langle\chi_{R}\left(A^{-1} F B^{-1} F^{\dagger}\right)\right\rangle_{\text {plain }} \\
& =\int[d F]_{n \times n}^{\mathbb{C}} e^{-\operatorname{tr}\left(F F^{\dagger}\right)} \chi_{R}\left(A^{-1} F B^{-1} F^{\dagger}\right) \\
& =\frac{|R| ! \chi_{R}\left(A^{-1}\right) \chi_{R}\left(B^{-1}\right)}{d_{R}} .
\end{aligned}
$$

For an $F$ model correlation function the vertices are dictated by a partition $\lambda$ of $k$, so that the $i_{p}(\lambda)$ parts of $\lambda$ of length $p$ correspond to $i_{p}(\lambda) 2 p$-valent vertices. Expanding with the formula (50)

$$
\begin{aligned}
& \left\langle\prod_{p=1}^{k}\left[\operatorname{tr}\left(A^{-1} F B^{-1} F^{\dagger}\right)^{p}\right]^{i_{p}(\lambda)}\right\rangle_{\text {plain }} \\
& =\left\langle\operatorname{tr}\left(\alpha_{\lambda}\left(A^{-1} F B^{-1} F^{\dagger}\right)^{\otimes k}\right)\right\rangle_{\text {plain }} \\
& =\sum_{R \vdash k} \frac{k ! \chi_{R}\left(\alpha_{\lambda}\right) \chi_{R}\left(A^{-1}\right) \chi_{R}\left(B^{-1}\right)}{d_{R}}
\end{aligned}
$$

Expanding out the characters $\chi_{R}\left(A^{-1}\right)$ and $\chi_{R}\left(B^{-1}\right)$ as multitraces gives

$$
\begin{aligned}
& \sum_{R \vdash k} \sum_{\mu, \nu \vdash k} \frac{|[\nu]||[\mu]|}{k !} \operatorname{tr}\left(\alpha_{\mu}\left(A^{-1}\right)^{\otimes k}\right) \operatorname{tr}\left(\alpha_{\nu}\left(B^{-1}\right)^{\otimes k}\right) \\
& \quad \times \frac{\chi_{R}\left(\alpha_{\lambda}\right) \chi_{R}\left(\alpha_{\mu}\right) \chi_{R}\left(\alpha_{\nu}\right)}{d_{R}} .
\end{aligned}
$$

This expansion is important because we see how the faces of the $F$ model diagrams are controlled by the partitions $\mu$ and $\nu$ that organize the couplings $\{t\}$ and $\{\bar{t}\}$. Comparing (52) to its $Z$ model equivalent (45) and picking out the 

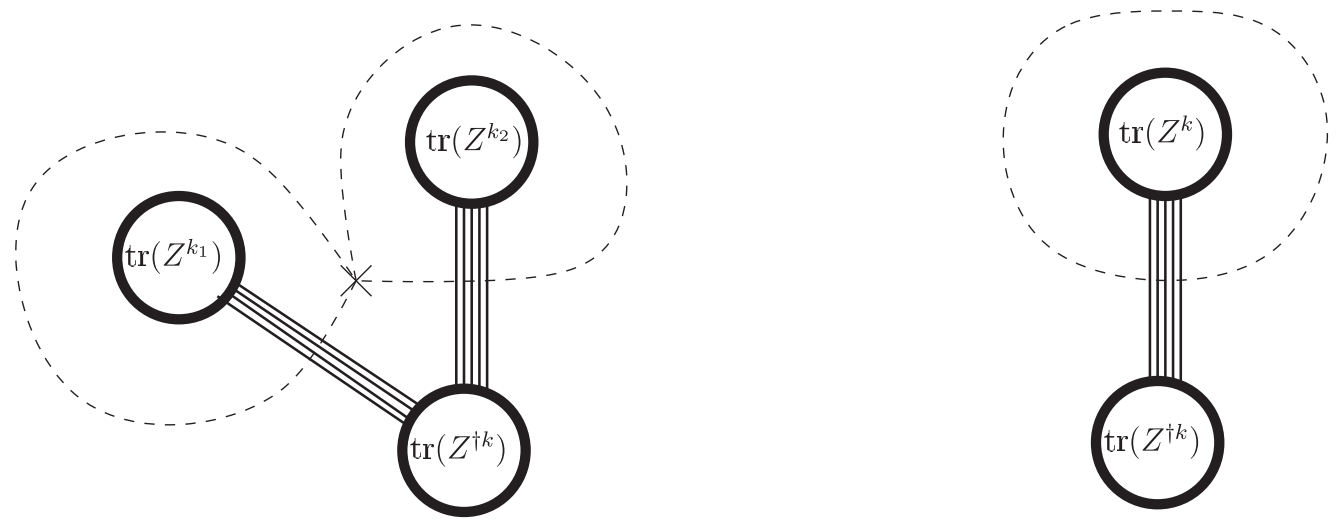

FIG. 8. $Z$ model three-point function and two-point function on the sphere with bunched propagators. The dual graph is drawn with a dashed line.

terms for particular face configurations in the respective models

$$
\begin{aligned}
& k !\left\langle\operatorname{tr}\left(\Sigma_{\lambda}\left(A^{-1} F B^{-1} F^{\dagger}\right)^{\otimes p}\right)\right\rangle_{\text {plain, } \mu, \nu \text { term }} \\
& =\left\langle\operatorname{tr}\left(\Sigma_{\nu} Z^{\dagger \otimes k}\right) \operatorname{tr}\left(\Sigma_{\mu} Z^{\otimes k}\right)\right\rangle_{\lambda \text { term }} \\
& =\sum_{R \vdash k} \frac{\chi_{R}\left(\Sigma_{\nu}\right) \chi_{R}\left(\Sigma_{\lambda}\right) \chi_{R}\left(\Sigma_{\mu}\right)}{d_{R}}=k ! \delta\left(\Sigma_{\nu} \Sigma_{\lambda} \Sigma_{\mu}\right) .
\end{aligned}
$$

In other words, both models are doing nothing other than computing the conjugacy class algebra of the symmetric group.

If we use the corrected propagator (23), then there are no 2 -valent vertices in the $F$ model, corresponding to the fact that all parallel propagators of the $Z$ model are bunched into single edges. Thus with the corrected propagator for the $F$ model the partitions $\lambda$ of vertices have no parts of length $1, i_{1}(\lambda)=0$. To relate this to the above analysis above define $\lambda^{r}=\lambda+\left[1^{r}\right]$ so that

$$
\begin{aligned}
& k !\left\langle\operatorname{tr}\left(\sum_{\lambda}\left(A^{-1} F B^{-1} F^{\dagger}\right)^{\otimes k}\right)\right\rangle \\
& =k ! \sum_{r=0}^{\infty}\left\langle\operatorname{tr}\left(\sum_{\lambda^{r}}\left(A^{-1} F B^{-1} F^{\dagger}\right)^{\otimes k+r}\right)\right\rangle_{\text {plain }} \\
& =\sum_{r=0}^{\infty} \sum_{\mu, \nu \vdash k+r} \operatorname{tr}\left(\alpha_{\mu}\left(A^{-1}\right)^{\otimes k+r}\right) \operatorname{tr}\left(\alpha_{\nu}\left(B^{-1}\right)^{\otimes k+r}\right) \\
& \quad \times\left\langle\operatorname{tr}\left(\Sigma_{\nu} Z^{\dagger \otimes k+r}\right) \operatorname{tr}\left(\Sigma_{\mu} Z^{\otimes k+r}\right)\right\rangle_{\Sigma_{\lambda^{k}} \text { term. }}
\end{aligned}
$$

Even if we fix the structure of $\mu, \nu, \lambda$ there are still an infinite number of $Z$ diagrams for every $F$ diagram because in the $Z$ model we can have any number of bunched parallel propagators at each edge, corresponding to adding arbitrary numbers of 2-valent vertices to the plain $F$ model.

In the original vanilla $Z$ model with $S=\mathbb{\rrbracket}_{N}$ picking a particular power of $N$ in the expansion of the correlation function (39) corresponds to fixing the genus of the diagrams in the $Z$ model expansion. Looking at the expansion of $\Omega_{k}$ in terms of cut-and-join operators $\Sigma_{\lambda}$ in Eq. (38), fixing the genus means we only consider $\lambda$ composed from a fixed number of transpositions $T([\lambda])=p$. The generic $\lambda$ with this property is $\left[2^{p}\right]$, followed by cases where the transpositions blend into longer cycles $\left[3,2^{p-2}\right],\left[4,2^{p-3}\right],\left[3,3,2^{p-4}\right]$, etc. For example, $[2,2] \rightarrow[3]$ corresponds to the degenerate multiplication of two transpositions when they share an element $i$ to get a 3 -cycle $(i k)(i j)=(i j k)$.

In the $F$ model $\lambda=\left[2^{p}\right]$ means $p$-valent vertices in the graph. The other terms for fixed genus, such as $\left[3,2^{p-2}\right]$, correspond to vertices in the $F$ model colliding to create higher-valency vertices. For example the collision of two 4 -valent vertices into a single 6-valency vertex for the dual to the $Z$ model torus two-point function in Fig. 9 is the counterpart of $[2,2] \rightarrow[3]$. The limit with only the generic cut-and-join operator $\Sigma_{\left[2^{p}\right]}$, corresponding to having only 4 -valent vertices in the $F$ model, is discussed in Sec. IV C.

Note that the number of edges $E$ in the $Z$ or $F$ model diagram is given by a sum over the faces/vertices weighted by their valency $2 E=\sum_{p=1}^{k} 2 p i_{p}(\lambda)=\sum_{p=1} p\left(i_{p}(\mu)+\right.$ $\left.i_{p}(\nu)\right)$. This is automatic since $\lambda, \mu$ and $\nu$ are each partitions of $E=k$.

Finally, to prove Eq. (30) for the full expansion of the $F$ model partition function expand the exponential using (48). With couplings $s_{k}=\frac{1}{k} \operatorname{tr}\left(S^{k}\right)$ to the $2 k$-valent vertices

$$
\begin{aligned}
& \int[d F]_{n \times n}^{\mathbb{C}} e^{-\operatorname{tr}\left(F F^{\dagger}\right)+\sum_{k=1}^{\infty} s_{k} \operatorname{tr}\left(A^{-1} F B^{-1} F^{\dagger}\right)^{k}} \\
& =\sum_{k=0}^{\infty} \sum_{\lambda \vdash k} \frac{|[\lambda]|}{k !} \operatorname{tr}\left(\alpha_{\lambda} S^{\otimes k}\right) \\
& \quad \times\left\langle\operatorname{tr}\left(\alpha_{\lambda}\left(A^{-1} F B^{-1} F^{\dagger}\right)^{\otimes k}\right)\right\rangle_{\text {plain }} \\
& =\sum_{k=0}^{\infty} \sum_{R \vdash k} \frac{|R| ! \chi_{R}(S) \chi_{R}\left(A^{-1}\right) \chi_{R}\left(B^{-1}\right)}{d_{R}} .
\end{aligned}
$$



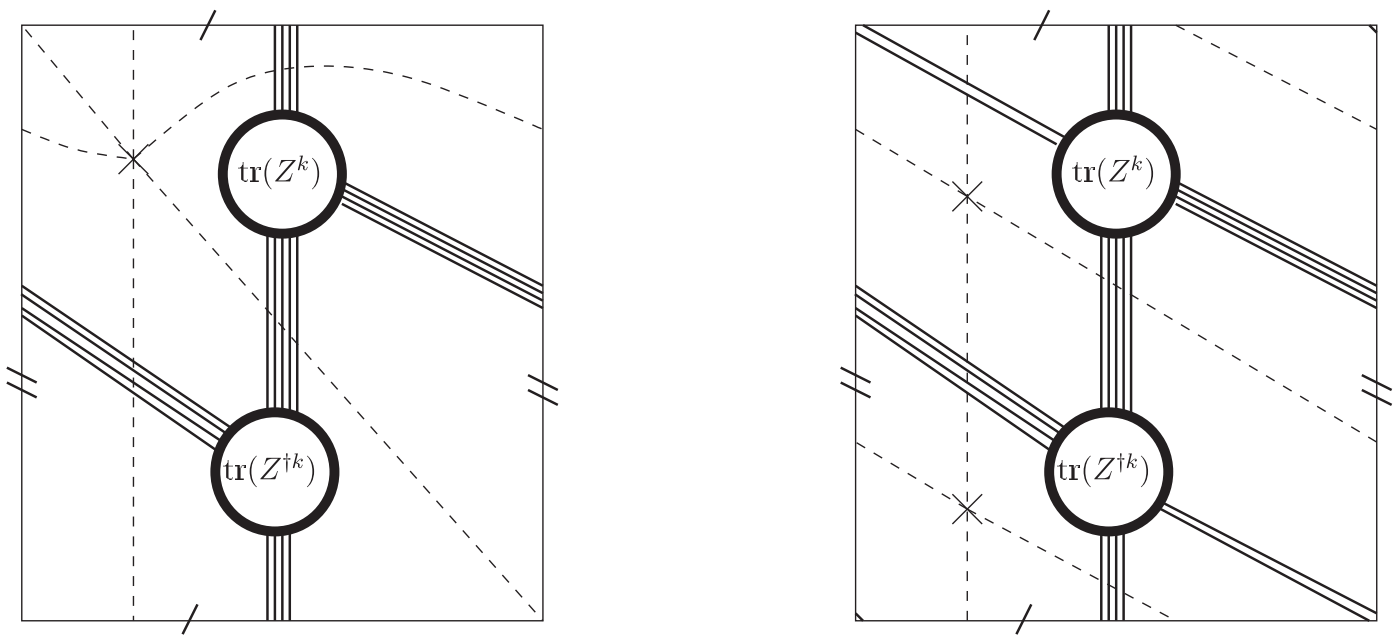

FIG. 9. The two different bunchings of propagators with no crossing for the $Z$ model two-point function on the torus: three bunchings from $\Sigma_{[3]}$ on the left and four bunchings from $\Sigma_{[2,2]}$ on the right. The dual graphs are drawn with dashed lines. The left dual graph can be considered as a degenerate case of the right graph when the two vertices of the dual graph on the right collide.

\section{A large $k$ limit}

It was shown in $[13,54]$ that if you take a given correlation function in the $Z$ model and fix the genus, corresponding to a particular power of $N$, then in the large $k$ limit of operators with many powers of $Z$ the cut-and-join operator $\Sigma_{\left[2^{p}\right]}$ dominates [cf. the torus two-point function in (A3)]

$$
\begin{aligned}
& \left\langle\operatorname{tr}\left(\alpha_{\nu} Z^{\dagger \otimes k}\right) \operatorname{tr}\left(\alpha_{\mu} Z^{\otimes k}\right)\right\rangle_{N^{k-p}} \text { term } \\
& =\frac{k ! N^{k}}{|[\mu]||[\nu]|} \sum_{\lambda: T([\lambda])=p} \frac{1}{N^{p}} \delta\left(\Sigma_{\nu} \Sigma_{\lambda} \Sigma_{\mu}\right) \\
& \stackrel{k \rightarrow \infty}{\rightarrow} \frac{k ! N^{k-p}}{|[\mu]||[\nu]|} \delta\left(\Sigma_{\nu} \Sigma_{\left[2^{p}\right]} \Sigma_{\mu}\right) .
\end{aligned}
$$

In other words, if we take the expansion of $\Omega_{k}$ in cut-andjoin operators in Eq. (38) and take this limit for each inverse power of $N$ then the $\Sigma_{\left[2^{p}\right]}$ term always dominates as a function of $k$ in the correlation functions at this genus

$$
\Omega_{k}=\sum_{p=0}^{k} \frac{1}{N^{p}} \sum_{\lambda:} \Sigma_{T(\lambda)=p} \stackrel{k \rightarrow \infty}{\rightarrow} \sum_{p=0}^{\infty} \frac{1}{N^{p}} \Sigma_{\left[2^{p}\right]} .
$$

For a fixed genus the terms we lose in this limit correspond to the degenerate cases where transpositions collide to give higher cycles. These degenerate cases appear with less frequency so they are suppressed for large $k$.

Since in this large $k$ limit $\Sigma_{\left[2^{p}\right]} \sim \frac{1}{p !} \Sigma_{[2]}^{p}, \Omega_{k}$ can be said to exponentiate $\Omega_{k} \rightarrow \exp \left(\frac{1}{N} \Sigma_{[2]}\right)$. The sum over transpositions $\Sigma_{[2]}$ has a simple interpretation in terms of either splitting or joining a trace. Geometrically this means that $n$ punctured genus $g$ surfaces factorize into 3-punctured spheres. See [13] for more details.

Here $N$ has been treated as a bookkeeping device for the genus. If we allow $N$ to be a number, then the limit must be taken delicately. Really we are taking the double-scaling BMN limit $k \sim N^{1 / 2} \rightarrow \infty$ [40] and expanding with the nonplanar coupling $g_{2}=\frac{k^{2}}{N}<1$, cf. [13]. If $k$ grows faster than $N^{1 / 2}$ then other terms become significant and spoil the simple exponentiation $\Omega_{k} \rightarrow \exp \left(\frac{1}{N} \Sigma_{[2]}\right)$.

In terms of the $F$ model, restricting to cut-and-join operators of the form $\Sigma_{\left[2^{p}\right]}$ means that we only have 4 -vertices in the model. In terms of the couplings $s_{k}$, we are setting $s_{k}=0$ for $k \geq 3$ and only retaining 4-vertices $s_{1}=N, s_{2}=\frac{N}{2}$. Diagrams involving higher-valency vertices resulting from collisions of 4-vertices are dropped.

In the $2 \mathrm{D}$ topological gravity case the Hermitian matrix model with couplings $\sum_{k=1} t_{k} \operatorname{tr}\left(M^{k}\right)$ can also be rearranged exactly into a Kontsevich-Penner model before taking the double-scaling limit. This is the Chekhov-Makeenko model [35] and is the Hermitian version of the complex matrix model duality discussed here. The dual model has vertices of all valency, including odd ones. Taking the double-scaling limit eliminates all but the 3 -valent terms, giving the standard Kontsevich model.

\section{Hurwitz theory for $\mathbb{C P}^{\mathbf{1}} \backslash\{\mathbf{0}, \infty\}$}

The formulae for the correlation functions as sums over cut-and-join operators (39) can be interpreted in terms of Hurwitz numbers that count holomorphic maps from the Riemann surface $\mathbb{S}_{g}$ on which a graph is drawn to the sphere $\mathbb{C P}^{1}$ with three branch points. For a $k$-sheeted covering of $\mathbb{C P}^{1}$ by a genus $g$ surface with ramification profiles $\mu, \nu$ and $\lambda$ the number of coverings is 


$$
\begin{aligned}
\operatorname{Cov}_{k}^{g}(\nu, \lambda, \mu) & :=\sum_{f(\nu, \lambda, \mu): \mathbb{S}_{g} \rightarrow \mathbb{C} \mathbb{P}^{1}} \frac{1}{|\operatorname{Aut}(f)|} \\
& =\frac{1}{k !} \delta\left(\Sigma_{\nu} \Sigma_{\lambda} \Sigma_{\mu}\right) .
\end{aligned}
$$

The maps are counted up to automorphisms of the covering map $f: \mathbb{S}_{g} \rightarrow \mathbb{C P} \mathbb{P}^{1}$. The genus $g$ for which this is nonvanishing is given by the Riemann-Hurwitz theorem, which relates the genus $g$ of $\mathbb{S}_{g}$ to the branching numbers at each branch point

$$
2 g-2=-2 k+T([\nu])+T([\lambda])+T([\mu]) .
$$

The branching number at each branch point is given by the minimum number of transpositions needed to build the conjugacy class corresponding to the partition. ${ }^{9}$ For our case this formula is just a restatement of the Euler characteristic formula (41).

So each correlation function is a sum over Hurwitz numbers for maps to $\mathbb{C} \mathbb{P}^{1}$ with three branch points

$$
\left\langle\operatorname{tr}\left(\Sigma_{\nu} Z^{\dagger \otimes k}\right) \operatorname{tr}\left(\Sigma_{\mu} Z^{\otimes k}\right)\right\rangle=(k !)^{2} \sum_{\lambda \vdash k} N^{C([\lambda])} \operatorname{Cov}_{k}(\nu, \lambda, \mu) .
$$

There is a similar story for the Hermitian matrix model $[23,56]$. The relation with the complex matrix model discussed here is simple: just replace one of the profiles $\nu$ with $\left[2^{k / 2}\right]$ to account for the Hermitian matrix model propagator. There is then an identity that comes from setting $\bar{t}_{k}=\delta_{k 2}$, known in the literature [23,31],

$$
\begin{aligned}
Z(\{t\},\{s\}) & =\int[d M]_{N \times N}^{H} e^{-\operatorname{tr}\left(S^{-1} M S^{-1} M\right)+\sum_{k=1}^{\infty} t_{k} \operatorname{tr}\left(M^{k}\right)} \\
& =\int[d Z]_{N \times N}^{\mathbb{C}} e^{-\operatorname{tr}\left(S^{-(1 / 2)} Z S^{-(1 / 2)} Z^{\dagger}\right)+\sum_{k=1}^{\infty} t_{k} \operatorname{tr}\left(Z^{k}\right)+\operatorname{tr}\left(Z^{\dagger 2}\right)} \\
& =\int[d F]_{N \times N}^{\complement} e^{-\operatorname{tr} F F^{\dagger}+\operatorname{tr}\left(A^{-1} F S F^{\dagger}\right)^{2}}
\end{aligned}
$$

In the expansion of the Hermitian model in Feynman diagrams, $k$-valent faces come with a coupling $s_{k}$, while $k$-valent vertices come with a coupling $t_{k}$ [23]. Graph duality in this model [5] just exchanges $\{t\}$ for $\{s\}$. The identity with the $Z$ model in the second line can be seen directly since the $\operatorname{tr}\left(Z^{\dagger 2}\right)$ term allows each propagator to loop back to the holomorphic operator built out of $Z$ 's, which is then equivalent to the Hermitian matrix model operator in the Hermitian correlation function. The difference in powers of $S$ in the actions arises because there are now two edges in the $Z$ model for every edge in the Hermitian model. The final line follows from the symmetry in the character expansion (29); the identity between the

\footnotetext{
${ }^{9}$ There is a review of Hurwitz theory in [52]. The relation of these cut-and-join operators and Hurwitz theory to integrable hierarchies is summarized in [55].
}

first and third line was proved in [23] using the same techniques as used in Sec. II. ${ }^{10}$

Taking the limit described in Sec. IVC so that $\Sigma_{\lambda} \rightarrow$ $\Sigma_{\left[2^{p}\right]} \sim \frac{1}{p !} \Sigma_{[2]}^{p}$, which for the $F$ model means restricting to only 4-valent vertices, the Hurwitz numbers become double Hurwitz numbers. Double Hurwitz numbers have two fixed branching profiles $\mu, \nu$ for branch points at 0 and $\infty$ and a remaining arbitrary number of simple branch points (with profile [2]). ${ }^{11}$ In this limit the partition function becomes the $\tau$ function for the Toda lattice hierarchy that appears in the Gromov-Witten theory of $\mathbb{C P}^{1}$ $[58,59]^{12}$

$$
\begin{aligned}
Z(\{t\},\{\bar{t}\},\{s\}) \rightarrow & \sum_{k=0}^{\infty} \sum_{\mu, \nu \vdash k} \sum_{p=0}^{\infty} \frac{s_{1}^{k-2 p}\left(2 s_{2}\right)^{p}}{k ! p !} \operatorname{tr}\left(\alpha_{\mu}\left(A^{-1}\right)^{\otimes k}\right) \\
& \times \operatorname{tr}\left(\alpha_{\nu}\left(B^{-1}\right)^{\otimes k}\right) \delta\left(\Sigma_{\mu} \Sigma_{[2]}^{p} \Sigma_{\nu}\right) \\
= & \sum_{k=0}^{\infty} \sum_{\mu, \nu \vdash k} \sum_{p=0}^{\infty} \frac{s_{1}^{k-2 p}\left(2 s_{2}\right)^{p}}{p !} \operatorname{tr}\left(\alpha_{\mu}\left(A^{-1}\right)^{\otimes k}\right) \\
& \times \operatorname{tr}\left(\alpha_{\nu}\left(B^{-1}\right)^{\otimes k}\right) \operatorname{Cov}_{k}(\mu, \nu, \underbrace{[2], \cdots[2]}_{p \text { times }}) .
\end{aligned}
$$

It should be possible to make this picture more precise: the $Z$ and $F$ models compute relative Gromov-Witten invariants for the topological $A$ model on $\mathbb{C P}^{1}$ with two points marked at 0 and $\infty$, cf. [60]. The holes in the $F$ world sheet corresponding to holomorphic $Z$ operators wrap around 0 , while the holes corresponding to antiholomorphic $Z^{\dagger}$ operators wrap around $\infty$. The parts of the cut-andjoin operator $\Sigma_{\lambda}$ then map to gravitational descendants $\tau_{\lambda_{i}}(w)$ of the Kähler class $w$. The full details of this correspondence require the technology of completed cycles [60].

The appearance of $\mathbb{C P} \mathbb{P}^{1} \backslash\{0, \infty\}$ is curious because of its appearance both as an auxiliary curve for the normal matrix model description of the $c=1$ string [14] and in the topological B model setup for the $c=1$ string in [61]. The free energy of the $c=1$ string at the self-dual radius is also known to agree with that for the topological A model on the conifold resolved by a sphere in the limit where the complexified Kähler class vanishes $t \rightarrow 0$ [2].

\footnotetext{
${ }^{10}$ Holomorphic maps onto $\mathbb{C P}^{1}$ with just three branch points are special. Belyi's theorem [57] states that a nonsingular Riemann surface is an algebraic curve defined over the algebraic numbers $\mathbb{Q}$ if and only if there is a holomorphic map of the Riemann surface onto $\mathbb{C} \mathbb{P}^{1}$ with only three branch points. For the Hermitian matrix model, where all the ramification orders over one of the branch points are 2, the Belyi map is a special type called "clean" [56].

${ }^{11}$ Single Hurwitz numbers occur when there is no branching at the second point, i.e. $\nu=\left[1^{k}\right]$. The generating function for single Hurwitz numbers is obtained from the $Z$ model with $\bar{t}_{k}=\delta_{k 1}$.

${ }^{12}$ The map to the variables in [59] is $p_{\mu}=\operatorname{tr}\left(\alpha_{\mu}\left(A^{-1}\right)^{\otimes k}\right)$, $p_{\nu}^{\prime}=\operatorname{tr}\left(\alpha_{\nu}\left(B^{-1}\right)^{\otimes k}\right), q=s_{1}$, and $\beta=\frac{2 s_{2}}{s_{1}^{2}}$.
} 


\section{THE $F$ MODEL AND THE CELL DECOMPOSITION OF $\mathcal{M}_{g, n}$}

The (Deligne-Mumford-compactified) moduli space $\mathcal{M}_{g, n}$ of Riemann surfaces of genus $g$ punctured $n$ times can be extended to the space of "ribbon" or "fat" graphs by replacing each puncture with a boundary of length $\ell_{i}$ for $i=1, \ldots n$. This extended decorated moduli space $\mathcal{M}_{g, n} \times \mathbb{R}_{+}^{n}$ has real dimension $6 g-6+3 n$. As discovered in the works of Mumford, Thurston, Strebel [62], Harer [63], Penner [34], and others, any point in this moduli space can be obtained by considering connected graphs with lengths assigned to each edge.

The Penner Hermitian matrix model [34]

$$
\int[d Q]^{H} e^{\operatorname{tr}(Q)+\operatorname{tr} \log (1-Q)}=\int[d Q]^{H} e^{-\sum_{k=2}^{\infty}(1 / k) \operatorname{tr}\left(Q^{k}\right)}
$$

gives a cell decomposition of $\mathcal{M}_{g, n} \times \mathbb{R}_{+}^{n}$. Each graph of the Penner model with $n$ faces and genus $g$ corresponds to one of the cells. The top-dimensional cells in $\mathcal{M}_{g, n} \times \mathbb{R}_{+}^{n}$ are swept out by the lengths of the $6 g-6+3 n$ edges of the Feynman graphs with only 3 -valent vertices. Lowerdimensional cells in the cell decomposition arise when we shrink an edge, colliding two vertices into a higher-valency vertex. Because of the extra factor of -1 from the vertices with each lowering of dimension, the Penner model calculates the virtual Euler characteristic of $\mathcal{M}_{g, n}$. The symmetry factors of the Feynman graphs account for the fact that $\mathcal{M}_{g, n}$ is an orbifold space.

Konstevich [3] adapted the 3-valent version of this model to give a generating function for the correlators of 2D topological gravity, which calculate intersection numbers on $\mathcal{M}_{g, n}$ [64]. The couplings $t_{n}$ to the operators are encoded in a matrix $Z$ by the transformation $t_{k}=\frac{1}{k} \operatorname{tr}\left(Z^{-k}\right)$. This constant matrix modifies the quadratic term in the Kontsevich matrix model

$$
\int[d M]^{H} e^{\operatorname{tr}\left(-(1 / 2) Z M^{2}+(1 / 6) M^{3}\right)} .
$$

In the expansion of this partition function, each Feynman graph with $n$ faces and genus $g$ can be written as an integral over the corresponding top-dimensional cell in $\mathcal{M}_{g, n} \times \mathbb{R}_{+}^{n}$ using the Schwinger parameterization of the propagators

$$
\left\langle M_{j}^{i} M_{l}^{k}\right\rangle=\delta_{l}^{i} \delta_{j}^{k} \frac{2}{z_{i}+z_{j}}=2 \delta_{l}^{i} \delta_{j}^{k} \int_{0}^{\infty} d p_{e} e^{-p_{e}\left(z_{i}+z_{j}\right)} .
$$

By integrating over the $6 g-6+3 n$ lengths $p_{e}$ of the edges of the graph the whole of the cell is covered. Separating out the integral over the boundary lengths $\mathbb{R}_{+}^{n}$ (which correspond to sums of the edges around each boundary) one is left with an integral over $\mathcal{M}_{g, n}$ corresponding to the closed string correlation function [3] (see $[38,65]$ for concise summaries).
The $F$ model only has vertices of even valency, which suggests that it localizes on lower-dimensional cells in the complex for $\mathcal{M}_{g, n} \times \mathbb{R}_{+}^{n}$. The maximal-dimensional cell for the $F$ model, corresponding to all vertices of valency 4 , has dimension $4 g-4+2 n$ corresponding to the number of edges in the diagrams. This localization on degenerate subspaces of the moduli space was noticed in $[66,67]$ when considering extremal four-point functions in $\mathcal{N}=4$ SYM.

Following the example of the Kontsevich-Penner Hermitian model of Chekhov and Makeenko [35] we will write each generic $F$ model graph as an integral over a discrete version of a lower-dimensional cell in $\mathcal{M}_{g, n} \times \mathbb{R}_{+}^{n}$ [39]. In the continuum limit the Chekhov-Makeenko model reduces to the Kontsevich model. In the continuum limit of the $F$ model we get a 4-valent model which is the complex analogue of the Kontsevich model.

With discretization parameter $\varepsilon$ rewrite $A=\sqrt{N} e^{\varepsilon L}$ and $B=\sqrt{N} e^{\varepsilon M}$, i.e. $a_{i}=\sqrt{N} e^{\varepsilon l_{i}}$ and $b_{j}=\sqrt{N} e^{\varepsilon m_{j}}$. The propagator (27) of the rescaled $F$ model (26) can then be written as

$$
\frac{1}{a_{i} b_{j}-N}=\frac{1}{N e^{\varepsilon l_{i}} e^{\varepsilon m_{j}}-N}=\frac{1}{N} \sum_{p=1}^{\infty} e^{-p \varepsilon\left(l_{i}+m_{j}\right)} .
$$

The sum is a discrete Schwinger parameterization of the edge length for the propagator. Each summand comes from an edge of the $F$ graph with integer length $p \varepsilon$, corresponding to a different number $p$ of bunched propagators in the dual $Z$ model graph, cf. (25). ${ }^{13}$ The integer length $\ell$ of the boundary of each face is the valency of the vertex of the dual $Z$ model graph, or in other words the power of the operator $\operatorname{tr}\left(Z^{\ell}\right)$ or $\operatorname{tr}\left(Z^{\dagger \ell}\right)$.

For an $F$ model graph with $V$ vertices, $E$ edges and faces corresponding to $Z$ vertices labeled by $f$ (and color index $i_{f}$ ) and $Z^{\dagger}$ faces labeled by $g$ (and color index $j_{g}$ ) the contribution is

$$
\begin{aligned}
& c N^{V} \sum_{\left\{i_{f}\right\},\left\{j_{g}\right\}} \prod_{E \text { edges }} \frac{1}{a_{i_{f}} b_{j_{g}}-N} \\
& =c N^{V-E} \sum_{\left\{i_{f}\right\},\left\{j_{g}\right\}} \prod_{E \text { edges }} \frac{1}{\varepsilon l^{\varepsilon l_{i}} e^{\varepsilon m_{j_{g}}}-1} \\
& =c N^{V-E} \sum_{\left\{i_{f}\right\},\left\{j_{g}\right\}} \prod_{r=1}^{E} \sum_{p_{r}=1}^{\infty} e^{-\varepsilon p_{r}\left(l_{i_{f}}+m_{j_{g}}\right)} .
\end{aligned}
$$

$c$ is the symmetry factor for the graph. The discrete sums for each of its $E$ propagators give a sum over discrete points in an $E$-dimensional cell of $\mathcal{M}_{g, n} \times \mathbb{R}_{+}^{n}$. Each point corresponds to a different $Z$ model graph with different

\footnotetext{
${ }^{13}$ Such a discrete metric on the moduli space also appears for the Hermitian matrix model in $[68,69]$, where the integer lengths also correspond to the number of bunched propagators between the vertices.
} 
numbers of bunched propagators between each set of vertices, given by the integers $p_{r}$. A given $Z$ model correlation function gets contributions from a finite number of graphs, so the closed string correlation function must localize on only a finite number of points in the moduli space.

The propagator in its discrete Schwinger parametrization (64) has a continuum limit as $\varepsilon \rightarrow 0$

$$
\lim _{\varepsilon \rightarrow 0} \sum_{p=1}^{\infty} \varepsilon e^{-p \varepsilon\left(l_{i}+m_{j}\right)}=\int_{0}^{\infty} d p e^{-p\left(l_{i}+m_{j}\right)}=\frac{1}{l_{i}+m_{j}} .
$$

The model with this propagator arises in a double-scaling limit of the $F$ model where we take $N$ large $N \sim \frac{1}{\varepsilon^{2}}$. Rescaling the $F$ model matrix $F=\sqrt{\epsilon} G$ the action (26) becomes

$$
\begin{aligned}
- & \operatorname{tr}\left(A F B F^{\dagger}\right)+N \sum_{k=1}^{\infty} \frac{1}{k} \operatorname{tr}\left(F F^{\dagger}\right)^{k} \\
= & -N \varepsilon \operatorname{tr}\left[\left(1+\varepsilon L+\mathcal{O}\left(\varepsilon^{2}\right)\right) G\left(1+\varepsilon M+\mathcal{O}\left(\varepsilon^{2}\right)\right) G^{\dagger}\right] \\
& +N \operatorname{tr}\left[\varepsilon G G^{\dagger}+\varepsilon^{2} \frac{1}{2} G G^{\dagger} G G^{\dagger}+\mathcal{O}\left(\varepsilon^{3}\right)\right] \\
= & -N \varepsilon^{2} \operatorname{tr}\left(G^{\dagger} L G\right)-N \varepsilon^{2} \operatorname{tr}\left(G M G^{\dagger}\right) \\
& +N \varepsilon^{2} \frac{1}{2} \operatorname{tr}\left(G G^{\dagger} G G^{\dagger}\right)+N \mathcal{O}\left(\varepsilon^{3}\right) \\
\rightarrow & -\operatorname{tr}\left(G^{\dagger} L G\right)-\operatorname{tr}\left(G M G^{\dagger}\right)+\frac{1}{2} \operatorname{tr}\left(G G^{\dagger} G G^{\dagger}\right) .
\end{aligned}
$$

This $G$ model is a complex analogue of the Kontsevich model (62)

$$
\int[d G]_{N \times N}^{\mathbb{C}} e^{-\operatorname{tr}\left(G^{\dagger} L G\right)-\operatorname{tr}\left(G M G^{\dagger}\right)+(1 / 2) \operatorname{tr}\left(G G^{\dagger} G G^{\dagger}\right)} .
$$

It has the propagator identified in (66), which is similar to that of the Kontsevich model (63)

$$
\left\langle G_{j}^{i} G_{l}^{\dagger k}\right\rangle=\delta_{l}^{i} \delta_{j}^{k} \frac{1}{l_{i}+m_{j}} .
$$

Looking at an individual Feynman graph of the $F$ model we can also see that only 4-valent graphs survive in this double-scaling limit. Suppose a graph has $i_{k}$ vertices of even valency $2 k$. There are $V=\sum_{k=2}^{\infty} i_{k}$ vertices in total and $E=\frac{1}{2} \sum_{k=2}^{\infty} 2 k i_{k}=\sum_{k=2}^{\infty} k i_{k}$ edges. Setting $N=\frac{1}{\varepsilon^{2}}$ and taking the limit $\varepsilon \rightarrow 0$ then the expression (65) is only nonvanishing if there is a factor of $\varepsilon$ for each edge [cf. (66)]

$$
N^{V-E}=\varepsilon^{E}
$$

This means that $E=2 V$, which is satisfied if only $i_{2}$ is nonzero. In this case we get

$$
\begin{gathered}
\lim _{\varepsilon \rightarrow 0} c \sum_{\left\{i_{f}\right\},\left\{j_{g}\right\}} \prod_{r=1}^{E}\left[\varepsilon \sum_{p_{r}=1}^{\infty} e^{-\varepsilon p_{r}\left(l_{i_{f}}+m_{j_{g}}\right)}\right] \\
=c \sum_{\left\{i_{f}\right\},\left\{j_{g}\right\}} \prod_{r=1}^{E} \int_{0}^{\infty} d p_{r} e^{-p_{r}\left(l_{i_{f}}+m_{j_{g}}\right)} \\
=c \sum_{\left\{i_{f}\right\},\left\{j_{g}\right\}} \prod_{E \text { edges }} \frac{1}{l_{i_{f}}+m_{j_{g}}} .
\end{gathered}
$$

This comes from the corresponding graph of the $G$ model.

The integrals over the world sheet boundary lengths $\mathbb{R}_{+}^{n}$ must be decoupled with care, since there is at least one relationship between the boundary lengths: the sum of the $Z$ boundary lengths must equal the sum of the $Z^{\dagger}$ boundary lengths. For example in Fig. 8 the three-point function has only two independent boundary lengths, not three. Once this is done, it is still not clear what quantity we are integrating over (a subspace of) $\mathcal{M}_{g, n}$.

The fact that the $G$ model has only 4-valent vertices relates it to the BMN limit from Sec. IV C, which arose from limiting the $F$ model to 4 -valent vertices. However it is still not clear what the $G$ model is calculating in this context. The obvious link would be if in the continuum limiting process the discrete sum only got contributions when $p \sim \frac{1}{\varepsilon}=\sqrt{N}$ (BMN-length operators propagating between vertices of the $Z$ model), but this is not the case. This issue is left for the future.

\section{CONCLUSIONS AND FUTURE DIRECTIONS}

In this paper we have studied correlation functions that correspond both to tachyon scattering for the $c=1, R=1$ noncritical string and to a half-BPS sector in free 4D $\mathcal{N}=4$ super-Yang-Mills. In the $Z$ complex matrix model the closed string insertions correspond to vertices of the Feynman diagrams. The $Z$ model is precisely dual to another complex matrix model called the $F$ model. In the $F$ model the closed string insertions are now associated to the faces of its Feynman diagrams. This duality can be shown using character expansions, or by integrating in and out fields to see the graph duality dynamically, following the program set out by Gopakumar [5].

Using the example of the Kontsevich model, the correlation functions of the $F$ model can be written as sums over discrete points in subspaces of the moduli space of punctured Riemann surfaces. These discrete points correspond to ribbon graphs with integer-length edges.

This complex matrix model duality could provide a prototype for understanding the AdS/CFT duality microscopically. It may be possible to rewrite (perhaps just free) $\mathcal{N}=4$ super Yang-Mills as a dual theory, where local 
operators and interaction vertices from $\mathcal{N}=4$ SYM correspond to faces of the dual Feynman graphs. The correlation functions of this dual theory would be easier to write as string moduli space integrals, following the Kontsevich schema. At nonzero coupling, summing over the interaction vertex holes should remove the D3 branes and alter the background to $\mathrm{AdS}_{5} \times S^{5}$.

There is a long way to go to realize this goal. In contrast to the program set out in [36-38], here we have dropped the spacetime dependence of the $\mathcal{N}=4$ correlation functions to focus on the combinatorial index structure from which the nonplanar expansion comes. Really the sector of $\mathcal{N}=4$ we have studied just computes a metric on multitrace half-BPS states and does not contain any spacetime information. More general correlation functions not only include more interesting spacetime dependence but should also get contributions from the full moduli space of punctured Riemann surfaces, not just subspaces.

As long as we keep the separation between holomorphic and antiholomorphic operators, it should be straightforward to introduce other complex scalar fields into this duality. ${ }^{14}$ The nonplanar expansion of the free theory in terms of cut-and-join operators discussed in Sec. IV follows through with little modification [13]. Allowing the scalars to be real, or introducing fermions and the gauge boson, would introduce more complication.

From the string side, it is important to understand how the reduction to the $c=1$ string from the full IIB string on $\mathrm{AdS}_{5} \times S^{5}$ works. Both contain a Liouville direction and it is tempting to identify the $R=1$ limit with the small radius limit of the bulk geometry corresponding to free SYM. The $c=1$ string in this limit is known to have various topological coset descriptions [71-73], so perhaps it is possible to use the cohomological reduction techniques of [74] for sigma models with supersymmetric target spaces to reduce the full bulk coset.

On the other hand, an alternative strategy would be to take a topological description of the $c=1, R=1$ string and try to include the full $P U(2,2 \mid 4)$ symmetry of free 4D $\mathcal{N}=4$ SYM. For example, it is known that the free energy of the $c=1, R=1$ string agrees with that for the $t \rightarrow 0$ limit of the topological $A$ model on the resolved conifold [2]. Once one understands how tachyon scattering is reproduced in that setting, one can think about how to include more of the spectrum of $\mathcal{N}=4$. An $A$ model approach seems promising given that the correlation functions were shown in Sec. IV D to count holomorphic maps onto $\mathbb{C} \mathbb{P}^{1}$. A tentative connection to the $A$ model on $\mathbb{C} \mathbb{P}^{1}$ was made at the end of that section.

Focusing now on the $F$ model there are several areas that merit further study:

\footnotetext{
${ }^{14}$ See Appendix D for a sketch of how this might work with two complex matrices.
}

(i) A brane interpretation of the $F$ model would be welcome, perhaps along the lines of the relation between the Kontsevich model and the open string field theory of FZZT branes for 2D topological gravity derived by Gaiotto and Rastelli [4]. Such an interpretation of the $W_{\infty}$ model has been discussed in [75,76].

(ii) What mechanism localizes the integral over the moduli space to discrete points in Sec. V? Given that this is also a feature of the Hermitian matrix model $[39,69]$, does it always arise in free theories?

(iii) The $Z$ model and the $F$ model capture tachyon scattering for the $c=1$ string at the self-dual radius, but they do not include all of the discrete states or the $S U(2)$ symmetry at this particular radius. Perhaps vortices appear in the $F$ model as holomorphic and antiholomorphic operators $\operatorname{tr}\left(F^{k}\right)$ and $\operatorname{tr}\left(F^{\dagger k}\right)$ like their appearance in the similar 6-vertex model [77].

(iv) The Toda integrable hierarchy structure of the $c=1$ string at the self-dual radius has not been discussed here from the point of view of the $F$ model.

(v) An algebraic geometry interpretation of the $F$ model might correspond to the limiting case discussed in [78] for the $c=1, R=1$ string.

(vi) The authors of [61] reproduced both the Kontsevich model and the $c=1, R=1$ tachyonic scattering matrix by considering noncompact branes in the topological B model in a deformed conifold background. A model similar to the $Z$ model was also studied in [79] for the $c=1, R=1$ string. Where does the $F$ model fit into this picture?

\section{ACKNOWLEDGMENTS}

We thank A. Alexandrov, R. de Mello Koch, R. Gopakumar, V. Kazakov, S. Ramgoolam, and V. Schomerus for discussions and comments on early drafts. We are also very grateful to the organizers of the Second Joburg Workshop on String Theory, during which many of these ideas were discussed.

\section{APPENDIX A: EXAMPLES FOR THE $F$ MODEL}

In this section we consider explicit examples of how the $F$ model Feynman diagrams reproduce the $Z$ model correlation functions. First we consider the $F$ model expansion.

A single 4-valent vertex $\left\langle\operatorname{tr}\left(F F^{\dagger} F F^{\dagger}\right)\right\rangle$ can only contract with itself to form a planar graph that is dual to the planar three-point function of the $Z$ model, cf. Figure 8 and Sec. A 1 for the full expansion.

For two 4-valent vertices $\left\langle\operatorname{tr}\left(F F^{\dagger} F F^{\dagger}\right) \operatorname{tr}\left(F F^{\dagger} F F^{\dagger}\right)\right\rangle$ there are several possible diagrams: 
(1) Each vertex entirely contracted with itself is disconnected and corresponds to two disconnected threepoint functions.

(2) Each vertex with just one self-contraction each corresponds to the lollipop planar $1 \rightarrow 3$ four-point function, see Sec. A 3.

(3) The planar diagram with each propagator connecting the two vertices corresponds to the planar $2 \rightarrow 2$ four-point function all connected in a loop, see Sec. A 4.

(4) The torus diagram with each propagator connecting the two vertices corresponds to the torus two-point function, see Sec. A 2.

All the cases for a single 6-valent vertex $\left\langle\operatorname{tr}\left(F F^{\dagger} F F^{\dagger} F F^{\dagger}\right)\right\rangle$ correspond to the collision of the two 4 -valent vertices considered above:

(1) One way of contracting it in a planar way with four faces corresponds to the $1 \rightarrow 3$ four-point function Y diagram, cf. Sec. A 3, that arises from colliding the two vertices from case 2. above.

(2) A topologically distinct way of contracting it in a planar way corresponds to the $2 \rightarrow 2$ degenerate $\left\langle\operatorname{tr}\left(Z^{p}\right) \operatorname{tr}\left(Z^{\dagger p+q}\right) \operatorname{tr}\left(Z^{\dagger q+r}\right) \operatorname{tr}\left(Z^{r}\right)\right\rangle$ from the collision of vertices in 3. above, cf. Sec. A 4.

(3) The vertex contracted with itself on the torus has two faces and is $\mathcal{M}_{1,2}$, cf. Sec. A 2 . This results from the collision of the vertices in 4 . above.

\section{1. $\mathcal{M}_{0,3}$}

The leading planar term of the three-point function in the $Z$ model is

$$
\left\langle\operatorname{tr}\left(Z^{\dagger k}\right) \operatorname{tr}\left(Z^{k_{1}}\right) \operatorname{tr}\left(Z^{k_{2}}\right)\right\rangle_{\text {sphere }}=k_{1} k_{2} k N^{k-1},
$$

where $k=k_{1}+k_{2}$. This corresponds to the cut-and-join operator $\Sigma_{[2]}$ which splits $\operatorname{tr}\left(Z^{\dagger k}\right)$ into two pieces, cf. the analysis in [13], where there are two bunches of homotopic propagators, see Fig. 8.

The dual graph, drawn in Fig. 8 has one 4-valent vertex, two propagators and three faces (which correspond to the old vertices). Reading the term in the rescaled $F$ matrix model (26)

$$
\begin{aligned}
\frac{N}{2}\left\langle\operatorname{tr}\left(F F^{\dagger} F F^{\dagger}\right)\right\rangle= & \frac{N}{2} \sum_{i_{1}, i_{2}, j_{1}, j_{2}}\left\langle F_{j_{1}}^{i_{1}} F^{\dagger j_{1}}{ }_{i_{2}} F_{j_{2}}^{i_{2}} F^{\dagger j_{2}}{ }_{i_{1}}\right\rangle \\
= & \frac{N}{2} \sum_{i_{1}, i_{2}, j_{1}, j_{2}}\left(\left\langle F_{j_{1}}^{i_{1}} F^{\dagger j_{1}}{ }_{i_{2}}\right\rangle\left\langle F_{j_{2}}^{i_{2}} F^{\dagger j_{2}}{ }_{i_{1}}\right\rangle\right. \\
& \left.+\left\langle F_{j_{1}}^{i_{1}} F^{\dagger j_{2}}{ }_{i_{1}}\right\rangle\left\langle F_{j_{2}}^{i_{2}} F^{\dagger j_{1}}{ }_{i_{2}}\right\rangle\right) .
\end{aligned}
$$

Note that there are no nonplanar terms in this correlator because of the configuration of the fields. ${ }^{15}$ Inserting the propagator (27) and then Taylor expanding each one

$$
\begin{aligned}
& \frac{N}{2} \sum_{i_{1}, i_{2}, j_{1}, j_{2}}\left(\frac{\delta_{i_{2}}^{i_{1}} \delta_{j_{1}}^{j_{1}}}{\left(a_{i_{1}} b_{j_{1}}-N\right)} \frac{\delta_{i_{1}}^{i_{2}} \delta_{j_{2}}^{j_{2}}}{\left(a_{i_{2}} b_{j_{2}}-N\right)}+\frac{\delta_{i_{1}}^{i_{1}} \delta_{j_{1}}^{j_{2}}}{\left(a_{i_{1}} b_{j_{2}}-N\right)} \frac{\delta_{i_{2}}^{i_{2}} \delta_{j_{2}}^{j_{1}}}{\left(a_{i_{2}} b_{j_{1}}-N\right)}\right) \\
& =\frac{N}{2} \sum_{i, j_{1}, j_{2}} \sum_{k_{1}, k_{2}=1}^{\infty} \frac{N^{k_{1}+k_{2}-2}}{a_{i}^{k_{1}+k_{2}} b_{j_{1}}^{k_{1}} b_{j_{2}}^{k_{2}}}+\sum_{i_{1}, i_{2}, j} \sum_{k_{1}, k_{2}=1}^{\infty} \frac{N^{k_{1}+k_{2}-2}}{a_{i_{1}}^{k_{1}} a_{i_{2}}^{k_{2}} b_{j}^{k_{1}+k_{2}}} \\
& =\frac{1}{2} \sum_{k_{1}, k_{2}=1}^{\infty}\left[\operatorname{tr}\left(A^{-k_{1}-k_{2}}\right) \operatorname{tr}\left(B^{-k_{1}}\right) \operatorname{tr}\left(B^{-k_{2}}\right)+\operatorname{tr}\left(A^{-k_{1}}\right) \operatorname{tr}\left(A^{-k_{2}}\right) \operatorname{tr}\left(B^{-k_{1}-k_{2}}\right)\right] N^{k_{1}+k_{2}-1} \\
& =\sum_{k_{1}<k_{2}}\left[t_{k_{1}} t_{k_{2}} \bar{t}_{k_{1}+k_{2}}+t_{k_{1}+k_{2}} \bar{t}_{k_{1}} \bar{t}_{k_{2}}\right] k_{1} k_{2}\left(k_{1}+k_{2}\right) N^{k_{1}+k_{2}-1}+\frac{1}{2 !} \sum_{k_{1}}\left[t_{k_{1}} t_{k_{1}} \bar{t}_{2 k_{1}}+t_{2 k_{1}} \bar{t}_{k_{1}} \bar{t}_{k_{1}}\right] 2 k_{1}^{3} N^{2 k_{1}-1} \text {. }
\end{aligned}
$$

This agrees with the expectation from (A1) where we get contributions from the conjugate correlation function too. Note that the generating function splits into two pieces depending on whether $k_{1}=k_{2}$, in which case we get a factorial from the exponential in the $Z$ action (1).

$$
\text { 2. } \mathcal{M}_{1,2}
$$

The torus two-point function for the $Z$ model is

$$
\begin{aligned}
\left\langle\operatorname{tr}\left(Z^{\dagger k}\right) \operatorname{tr}\left(Z^{k}\right)\right\rangle_{\text {torus }} & =\left\langle k\left|\left(\Sigma_{[3]}+\Sigma_{[2,2]}\right)\right| k\right\rangle N^{k-2} \\
& =k\left[\left(\begin{array}{c}
k \\
3
\end{array}\right)+\left(\begin{array}{c}
k \\
4
\end{array}\right)\right] N^{k-2} .
\end{aligned}
$$

Here we have used the cut-and-join notation of [13]. The different cut-and-join operators correspond to bunching homotopic propagators into either three or four bunches, cf. Fig. 9 for the two possibilities.

The bunching of the propagators into three, the left-hand diagram in Fig. 9, yields a dual graph with a single 6-valent vertex, three edges, and two faces. Reading the appropriate term in the $F$ matrix model we compute the nonplanar torus term for the 6 -valent vertex

\footnotetext{
${ }^{15}$ In a Gaussian Hermitian matrix model $\left\langle\operatorname{tr}\left(M^{4}\right)\right\rangle$ does however receive nonplanar contributions.
} 


$$
\begin{aligned}
& \frac{N}{3}\left\langle\operatorname{tr}\left(F F^{\dagger} F F^{\dagger} F F^{\dagger}\right)\right\rangle_{\text {torus }} \\
& \left.=\frac{N}{3} \sum_{i_{1}, i_{2}, i_{3}, j_{1}, j_{2}, j_{3}}\left\langle F_{j_{1}}^{i_{1}} F^{\dagger j_{1}}{ }_{i_{2}} F_{j_{2}}^{i_{2}} F^{\dagger j_{2}}{ }_{i_{3}} F_{j_{3}}^{i_{3}} F^{\dagger j_{3}}\right\rangle_{i_{1}}\right\rangle_{\text {torus }} \\
& =\frac{N}{3} \sum_{i_{1}, i_{2}, i_{3}, j_{1}, j_{2}, j_{3}}\left\langle F_{j_{1}}^{i_{1}} F^{\dagger j_{2}}{ }_{i_{3}}\right\rangle\left\langle F_{j_{3}}^{i_{3}} F^{\dagger j_{1}}{ }_{i_{2}}\right\rangle\left\langle F_{j_{2}}^{i_{2}} F^{\dagger j_{3}}{ }_{i_{1}}\right\rangle \\
& =\frac{N}{3} \sum_{i_{1}, i_{2}, i_{3}, j_{1}, j_{2}, j_{3}} \frac{\delta_{i_{3}}^{i_{1}} \delta_{j_{1}}^{j_{2}}}{\left(a_{i_{1}} b_{j_{1}}-N\right)} \frac{\delta_{i_{2}}^{i_{3}} \delta_{j_{3}}^{j_{1}}}{\left(a_{i_{3}} b_{j_{3}}-N\right)} \frac{\delta_{i_{1}}^{i_{2}} \delta_{j_{2}}^{j_{3}}}{\left(a_{i_{2}} b_{j_{2}}-N\right)} \\
& =\frac{N}{3} \sum_{i, j} \frac{1}{\left(a_{i} b_{j}-N\right)^{3}} \text {. }
\end{aligned}
$$

Now Taylor expand, using the binomial theorem

$$
\begin{aligned}
\frac{N}{3} \sum_{i, j} \sum_{k=3}^{\infty}\left(\begin{array}{c}
k-1 \\
2
\end{array}\right) \frac{N^{k-3}}{a_{i}^{k} b_{j}^{k}}= & \frac{1}{3} \sum_{k=3}^{\infty}\left(\begin{array}{c}
k-1 \\
2
\end{array}\right) \operatorname{tr}\left(A^{-k}\right) \\
& \times \operatorname{tr}\left(B^{-k}\right) N^{k-2} \\
= & \sum_{k=3}^{\infty} k\left(\begin{array}{l}
k \\
3
\end{array}\right) t_{k} \bar{t}_{k} N^{k-2}
\end{aligned}
$$

This agrees with the expectation from (A5).

The bunching of the propagators into four, the righthand diagram in Fig. 9, yields a dual graph with two 4-valent vertices, four edges, and two faces ${ }^{16}$ :

$$
\begin{aligned}
& \frac{N^{2}}{2 \cdot 2 \cdot 2 !}\left\langle\operatorname{tr}\left(F F^{\dagger} F F^{\dagger}\right) \operatorname{tr}\left(F F^{\dagger} F F^{\dagger}\right)\right\rangle_{\text {torus }} \\
& =\frac{N^{2}}{8} \sum_{i_{1}, i_{2}, i_{3}, i_{4}, j_{1}, j_{2}, j_{3}, j_{4}} \\
& \times\left\langle F_{j_{1}}^{i_{1}} F^{\dagger j_{1}}{ }_{i_{2}} F_{j_{2}}^{i_{2}} F^{\dagger j_{2}}{ }_{i_{1}} F_{j_{3}}^{i_{3}} F^{\dagger j_{3}}{ }_{i_{4}} F_{j_{4}}^{i_{4}} F_{i_{3}}^{\dagger j_{4}}\right\rangle_{\text {torus }} .
\end{aligned}
$$

There are two relevant nonplanar contractions

$$
\begin{aligned}
& \left\langle F_{j_{1}}^{i_{1}} F^{\dagger j_{3}}{ }_{i_{4}}\right\rangle\left\langle F_{j_{2}}^{i_{2}} F^{\dagger j_{4}}{ }_{i_{3}}\right\rangle\left\langle F_{j_{3}}^{i_{3}} F^{\dagger j_{2}}{ }_{i_{1}}\right\rangle\left\langle F_{j_{4}}^{i_{4}} F^{\dagger j_{1}}{ }_{i_{2}}\right\rangle \\
& \quad+\left\langle F_{j_{1}}^{i_{1}} F^{\dagger j_{4}}{ }_{i_{3}}\right\rangle\left\langle F_{j_{2}}^{i_{2}} F^{\dagger j_{3}}{ }_{i_{4}}\right\rangle\left\langle F_{j_{3}}^{i_{3}} F^{\dagger j_{1}}{ }_{i_{2}}\right\rangle\left\langle F_{j_{4}}^{i_{4}} F^{\dagger j_{2}}{ }_{i_{1}}\right\rangle .
\end{aligned}
$$

These both give the same contribution so we get

$$
\begin{aligned}
\frac{N^{2}}{4} \sum_{i, j} \frac{1}{\left(a_{i} b_{j}-N\right)^{4}} & =\frac{N^{2}}{4} \sum_{i, j} \sum_{k=4}^{\infty}\left(\begin{array}{c}
k-1 \\
3
\end{array}\right) \frac{N^{k-4}}{a_{i}^{k} b_{j}^{k}} \\
& =\frac{1}{4} \sum_{k=4}^{\infty}\left(\begin{array}{c}
k-1 \\
3
\end{array}\right) \operatorname{tr}\left(A^{-k}\right) \operatorname{tr}\left(B^{-k}\right) N^{k-2} \\
& =\sum_{k=4}^{\infty} k\left(\begin{array}{l}
k \\
4
\end{array}\right) k 4 t_{k} \bar{t}_{k} N^{k-2} .
\end{aligned}
$$

\footnotetext{
${ }^{16}$ One might worry about disconnected nonplanar graphs where each 4-vertex only contracts with itself. Fortunately this diagram is not possible, cf. the $\mathcal{M}_{0,3}$ example.
}

\section{3. $\mathcal{M}_{0,4} 1 \rightarrow 3$}

The leading planar term of the $1 \rightarrow 3$ four-point function in the $Z$ model is

$$
\left\langle\operatorname{tr}\left(Z^{\dagger k}\right) \operatorname{tr}\left(Z^{k_{1}}\right) \operatorname{tr}\left(Z^{k_{2}}\right) \operatorname{tr}\left(Z^{k_{2}}\right)\right\rangle_{\text {sphere }}=k_{1} k_{2} k_{3} k(k-1) N^{k-2}
$$

where $k=k_{1}+k_{2}+k_{3}$. This result is made up from contributions from the two cut-and-join operators $\Sigma_{[2,2]}$ and $\Sigma_{[3]}$, corresponding to the lollipop diagram with four edges and the $\mathrm{Y}$ diagram with three edges, as explained in $[13,66]$. The lollipop comes from two 4-vertices of the $F$ model with a self-contraction each. The Y diagram comes from one of the planar ways of contracting a single 6 -vertex of the $F$ model.

\section{4. $\mathcal{M}_{0,4} 2 \rightarrow 2$}

For $k_{1}+k_{2}=k_{3}+k_{4}$ and $\max \left\{k_{i}\right\}=k_{1}$ the planar connected $2 \rightarrow 2$ four-point function is

$$
\begin{aligned}
& \left\langle\operatorname{tr}\left(Z^{\dagger k_{1}}\right) \operatorname{tr}\left(Z^{\dagger k_{2}}\right) \operatorname{tr}\left(Z^{k_{3}}\right) \operatorname{tr}\left(Z^{k_{4}}\right)\right\rangle_{\text {planar, connected }} \\
& \quad=k_{1} k_{2} k_{3} k_{4}\left(k_{1}-1\right) N^{k_{1}+k_{2}-2}
\end{aligned}
$$

For generic values of the $\left\{k_{i}\right\}$ the $Z$ graph has four bunched edges corresponding to $\Sigma_{[2,2]}$ and the dual graph is the planar contraction of two 4-vertices of the $F$ model with no self-contractions. In a degenerate case where $k_{1}-k_{3}=$ $k_{2}-k_{4}$ there are only three edges in the $Z$ model skeleton graph corresponding to $\Sigma_{[3]}$ and the dual graph has a single 6-valent vertex.

Note that this correlation function also receives contributions at order $N^{k_{1}+k_{2}}$ and $N^{k_{1}+k_{2}-2}$ if $k_{1}=k_{3}$ from disconnected two-point functions.

\section{5. $\mathcal{M}_{0,2}$}

The $F$ model diagram dual to the planar two-point function requires special treatment. The dual graph (see the right-hand side of Fig. 8) must be take using the plain Gaussian propagator (24) with the quadratic interaction terms in the faces between the parallel propagators of the $Z$ model. The $F$ model diagram corresponds to taking these quadratic interaction terms daisy chained with no selfcontractions

$$
\begin{aligned}
& \frac{N^{k}}{k !}\left\langle\left[\operatorname{tr}\left(A^{-1} F B^{-1} F^{\dagger}\right)\right]^{k}\right\rangle_{\text {plain, daisy }} \\
& \left.\quad=\frac{N^{k}}{k !}\left\langle\prod_{p=1}^{k} \sum_{i_{p}, j_{p}} \frac{1}{a_{i_{p}}} \frac{1}{b_{j_{p}}} F_{j_{p}}^{i_{p}} F^{\dagger j_{p}}\right\rangle_{i_{p}}\right\rangle_{\text {plain, daisy }} \\
& \quad=\frac{N^{k}}{k !}(k-1) ! \prod_{p=1}^{k} \sum_{i_{p}, j_{p}} \frac{1}{a_{i_{p}}} \frac{1}{b_{j_{p}}}\left\langle F_{j_{p}}^{i_{p}} F^{\left.\dagger j_{p+1}{ }_{i_{p+1}}\right\rangle_{\text {plain }} .}\right.
\end{aligned}
$$


Here $i_{k+1} \equiv i_{1}$ and similarly for $j_{k+1}$. There are $(k-1)$ ! completely equivalent ways of daisy chaining the Wick contractions. Now inserting the plain propagator (24)

$$
\begin{aligned}
\frac{N^{k}}{k} \prod_{p=1}^{k} \sum_{i_{p}, j_{p}} \frac{1}{a_{i_{p}}} \frac{1}{b_{j_{p}}} \delta_{i_{p+1}}^{i_{p}} \delta_{j_{p}}^{j_{p+1}} & =\frac{N^{k}}{k} \sum_{i, j} \frac{1}{a_{i}^{k}} \frac{1}{b_{j}^{k}}=k N^{k} t_{k} \bar{t}_{k} \\
& =t_{k} \bar{t}_{k}\left\langle\operatorname{tr}\left(Z^{k}\right) \operatorname{tr}\left(Z^{\dagger k}\right)\right\rangle_{\text {sphere }}
\end{aligned}
$$

In this final step we have used the formula for the planar two-point function.

Note that because we are using the plain version of the $F$ model there is no sum over topologically identical $Z$ graphs with bunched propagators; here the $Z$ correlation functions must be calculated separately for each $k$.

\section{APPENDIX B: EXAMPLES FOR THE $C, D$ MODEL}

In this section are calculations for the $C, D$ model (15) with quartic vertex and propagator (16).

\section{1. $\mathcal{M}_{0,3}$}

For the specific three-point function

$$
\left\langle\operatorname{tr}(Z) \operatorname{tr}(Z) \operatorname{tr}\left(Z^{\dagger 2}\right)\right\rangle_{\text {sphere }}=2 N
$$

exactly one $C, D$ diagram contributes. This diagram is the same as in Fig. 6(c) except that there are only two quartic vertices. Proceeding with Einstein summation on $e_{k}, f_{k}=$ $1, \cdots N$ only

$$
\begin{aligned}
& \left.\frac{1}{2 !} \sum_{i_{1}, i_{2} ; j_{1}, j_{2}}\left\langle C_{e_{1} i_{1}} C_{f_{1} i_{1}}^{\dagger} D_{f_{1} j_{1}} D_{e_{1} j_{1}}^{\dagger} C_{e_{2} i_{2}} C_{f_{2} i_{2}}^{\dagger} D_{f_{2} j_{2}} D_{e_{2} j_{2}}^{\dagger}\right\rangle\right|_{Z, Z, Z^{\dagger 2}} \\
& =\frac{1}{2} \sum_{i_{1}, i_{2} ; j_{1}, j_{2}}\left\langle C_{e_{1} i_{1}} C_{f_{1} i_{1}}^{\dagger}\right\rangle\left\langle D_{f_{1} j_{1}} D_{e_{2} j_{2}}^{\dagger}\right\rangle\left\langle D_{e_{1} j_{1}}^{\dagger} D_{f_{2} j_{2}}\right\rangle\left\langle C_{e_{2} i_{2}} C_{f_{2} i_{2}}^{\dagger}\right\rangle \\
& =\frac{1}{2} \sum_{i_{1}, i_{2} ; j_{1}, j_{2}} \frac{\delta_{e_{1} f_{1}} \delta_{i_{1} i_{1}}}{a_{i_{1}}} \frac{\delta_{f_{1} e_{2}} \delta_{j_{1} j_{2}}}{b_{j_{1}}} \frac{\delta_{e_{1} f_{2}} \delta_{j_{1} j_{2}}}{b_{j_{1}}} \frac{\delta_{e_{2} f_{2}} \delta_{i_{2} i_{2}}}{a_{i_{2}}} \\
& =\frac{1}{2} \sum_{i_{1}, i_{2} ; j} \frac{1}{a_{i_{1}}} \frac{1}{a_{i_{2}}} \frac{1}{b_{j}^{2}} N=\frac{1}{2 !} t_{1} t_{1} \bar{t}_{2} 2 N .
\end{aligned}
$$

\section{2. $\mathcal{M}_{1,2}$}

For the specific torus two-point function

$$
\left\langle\operatorname{tr}\left(Z^{3}\right) \operatorname{tr}\left(Z^{\dagger 3}\right)\right\rangle_{\text {torus }}=3 N,
$$

the relevant torus $C, D$ diagram has three quartic vertices

$$
\begin{gathered}
\frac{1}{3 !} \sum_{\left\{i_{k}\right\},\left\{j_{k}\right\}}\left\langle C_{e_{1} i_{1}} C_{f_{1} i_{1}}^{\dagger} D_{f_{1} j_{1}} D_{e_{1} j_{1}}^{\dagger} C_{e_{2} i_{2}} C_{f_{2} i_{2}}^{\dagger} D_{f_{2} j_{2}} D_{e_{2} j_{2}}^{\dagger}\right. \\
\left.\times C_{e_{3} i_{3}} C_{f_{3} i_{3}}^{\dagger} D_{f_{3} j_{3}} D_{e_{3} j_{3}}^{\dagger}\right\rangle_{\text {torus }} .
\end{gathered}
$$

There are two ways of Wick contracting; one choice gives half of the result

$$
\begin{aligned}
& \frac{1}{3 !} \sum_{\left\{i_{k}\right\},\left\{j_{k}\right\}}\left\langle C_{e_{1} i_{1}} C_{f_{2} i_{2}}^{\dagger}\right\rangle\left\langle C_{f_{1} i_{1}}^{\dagger} C_{e_{3} i_{3}}\right\rangle\left\langle D_{f_{1} j_{1}} D_{e_{2} j_{2}}^{\dagger}\right\rangle\left\langle D_{e_{1} j_{1}}^{\dagger} D_{f_{3} j_{3}}\right\rangle \\
& \quad \times\left\langle C_{e_{2} i_{2}} C_{f_{3} i_{3}}^{\dagger}\right\rangle\left\langle D_{f_{2} j_{2}} D_{e_{3} j_{3}}^{\dagger}\right\rangle=\frac{1}{3 !} \sum_{i, j} \frac{1}{a_{i}^{3}} \frac{1}{b_{j}^{3}} N=\frac{1}{2} t_{3} \bar{t}_{3} 3 N .
\end{aligned}
$$

\section{APPENDIX C: RELATION OF THE $F$ MODEL TO THE $W_{\infty}$ MODEL}

In this section we show that the $W_{\infty}$ model [41,42] can be directly related to the $F$ model by a change of variables. ${ }^{17}$ Take the $W_{\infty}$ model with $\nu=-i \mu=N$, so that the $\log M$ term is tuned away, and expand the exponentiated operators in the same way as in Eq. (48)

$$
\begin{aligned}
& \int {[d M]_{N \times N}^{H^{+}} e^{-\operatorname{tr}(M)+\sum_{k=1}^{\infty} \bar{t}_{k} \operatorname{tr}\left[\left(M A^{-1}\right)^{k}\right]} } \\
&=\int[d M] e^{-\operatorname{tr}(M)} \sum_{l(R) \leq N} \chi_{R}\left(B^{-1}\right) \chi_{R}\left(M A^{-1}\right) .
\end{aligned}
$$

The eigenvalues of $M$ must be positive semidefinite for this integral to be well defined. Shortly we will see how this condition is automatically implemented by the $F$ model. Make the change of variables $M=U D U^{\dagger}$ where $U$ is unitary and $D$ is diagonal with eigenvalues $m_{1}, \ldots m_{N} \geq 0$

$$
\begin{aligned}
& \int[d U] \prod_{i=1}^{N} d m_{i} \Delta^{2}\left(m_{i}\right) e^{-\operatorname{tr}(D)} \sum_{l(R) \leq N} \chi_{R}\left(B^{-1}\right) \chi_{R}\left(U D U^{\dagger} A^{-1}\right) \\
& =\int \prod_{i=1}^{N} d m_{i} \Delta^{2}\left(m_{i}\right) e^{-\operatorname{tr}(D)} \sum_{l(R) \leq N} \chi_{R}\left(B^{-1}\right) \\
& \quad \times \frac{\chi_{R}(D) \chi_{R}\left(A^{-1}\right)}{\operatorname{dim}_{N} R} .
\end{aligned}
$$

$\Delta\left(m_{i}\right)$ is the standard Vandermonde determinant. In the final line we have used the integral [53]

$$
\int[d U]_{N \times N}^{U} \chi_{R}\left(U X U^{\dagger} Y\right)=\frac{\chi_{R}(X) \chi_{R}(Y)}{\operatorname{dim}_{N} R} .
$$

We will now manipulate the $F$ model in a similar way to get the same answer (C2). The complex matrix $F$ can be written with two unitary matrices $U, W$ and a diagonal matrix $D$ [22]

$$
F=W \sqrt{D} U^{\dagger}, \quad F^{\dagger}=U \sqrt{D} W^{\dagger} .
$$

The eigenvalues $m_{1}, \ldots m_{n} \geq 0$ of the diagonal matrix $D$ are the real, non-negative eigenvalues of $F F^{\dagger}$. The measure is then

\footnotetext{
${ }^{17}$ The proof in this section was carried out in collaboration with Hanna Grönqvist of the University of Helsinki.
} 


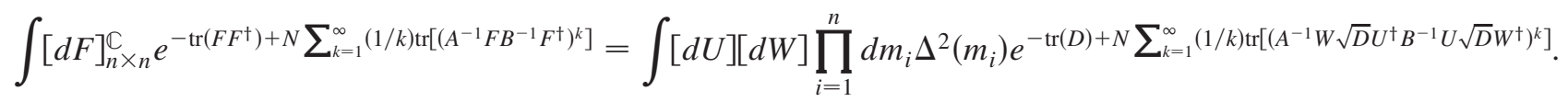

Character expanding the exponential with (48) and using (C3) on the $[d W]$ integral

$$
\begin{gathered}
\int[d U][d W] \prod_{i=1}^{n} d m_{i} \Delta^{2}\left(m_{i}\right) e^{-\operatorname{tr}(D)} \sum_{l(R) \leq N} \chi_{R}\left(\rrbracket_{N}\right) \chi_{R}\left(A^{-1} W \sqrt{D} U^{\dagger} B^{-1} U \sqrt{D} W^{\dagger}\right) \\
=\int[d U] \prod_{i=1}^{n} d m_{i} \Delta^{2}\left(m_{i}\right) e^{-\operatorname{tr}(D)} \sum_{l(R) \leq N} \operatorname{dim}_{N} R \frac{\chi_{R}\left(A^{-1}\right) \chi_{R}\left(D U^{\dagger} B^{-1} U\right)}{\operatorname{dim}_{n} R} .
\end{gathered}
$$

Next use (C3) on the $[d U]$ integral

$$
\int \prod_{i=1}^{n} d m_{i} \Delta^{2}\left(m_{i}\right) e^{-\operatorname{tr}(D)} \sum_{l(R) \leq N} \frac{\operatorname{dim}_{N} R \chi_{R}\left(A^{-1}\right) \chi_{R}(D) \chi_{R}\left(B^{-1}\right)}{\operatorname{dim}_{n} R \operatorname{dim}_{n} R}
$$

This already agrees with (C2) if we choose $n=N$. For the case $n \geq N$, compare (C7) with the character expansion of the $F$ model (29) to see that

$$
\int \prod_{i=1}^{n} d m_{i} \Delta^{2}\left(m_{i}\right) e^{-\operatorname{tr}(D)} \chi_{R}(D)=\left[\operatorname{dim}_{n} R\right]^{2}
$$

Inserting this into (C2) we get agreement with (29).

\section{APPENDIX D: COMPLEX MATRIX MODEL DUALITY FOR TWO (OR MORE)}

In this section we sketch how the duality might work for a $V$-type model with two $N \times N$ complex matrices $X, Y$, corresponding to two of the three complex scalars of free $4 \mathrm{D} \mathcal{N}=4 \mathrm{SYM}$ :

$$
\int[d X]^{\mathbb{C}}[d Y]^{\mathbb{C}} e^{-\operatorname{tr}\left(X X^{\dagger}\right)-\operatorname{tr}\left(Y Y^{\dagger}\right)+} \sum_{\mu_{1}, \mu_{2},[\alpha]} t_{\left\{\mu_{1}, \mu_{2},[\alpha]\right\}} \operatorname{tr}\left(\alpha X^{\mu_{1}} Y^{\left.\mu_{2}\right)}+\sum_{\mu_{1}, \mu_{2},[\alpha]} \bar{t}_{\left\{\mu_{1}, \mu_{2},[\alpha]\right\}} \operatorname{tr}\left(\alpha X^{\left.\dagger \mu_{1} Y^{\dagger \mu_{2}}\right)} .\right.\right.
$$

The sum is over all the holomorphic single-trace operators built out of $\mu_{1} X$ 's and $\mu_{2} Y^{\prime}$ 's. $\alpha$ is a single $k$ cycle $\alpha \in$ $[k] \subset S_{\mu_{1}+\mu_{2}}$ where $k=\mu_{1}+\mu_{2}$. The trace with a permutation is defined by

$$
\operatorname{tr}\left(\alpha X^{\mu_{1}} Y^{\mu_{2}}\right)=X_{i_{\alpha(1)}}^{i_{1}} \cdots X_{i_{\alpha\left(\mu_{1}\right)}}^{i_{\mu_{1}}} Y_{i_{\alpha\left(\mu_{1}+1\right)}}^{i_{\mu_{1}+1}} \cdots Y_{i_{\alpha\left(\mu_{1}+\mu_{2}\right)}}^{i_{\mu_{1}+\mu_{2}}}
$$

It is unique up to conjugation $\alpha \sim \rho^{-1} \alpha \rho$ for $\rho \in S_{\mu_{1}} \times S_{\mu_{2}}$ so we only sum over conjugacy classes [ $\alpha$ ] for this relation. The couplings $t, \bar{t}$ can be encoded in a generalized Kontsevich-Miwa transformation

$$
t_{\left\{\mu_{1}, \mu_{2},[\alpha]\right\}}=\frac{1}{\left|\operatorname{Sym}(\alpha) \cap S_{\mu_{1}} \times S_{\mu_{2}}\right|} \operatorname{tr}\left(\alpha A^{\mu_{1}} C^{\mu_{2}}\right), \quad \bar{t}_{\left\{\mu_{1}, \mu_{2},[\alpha]\right\}}=\frac{1}{\left|\operatorname{Sym}(\alpha) \cap S_{\mu_{1}} \times S_{\mu_{2}}\right|} \operatorname{tr}\left(\alpha B^{\mu_{1}} D^{\mu_{2}}\right) .
$$

The matrices $A, B, C, D$ do not commute and are not diagonalizable, unlike the single complex matrix case. For a single cycle $\operatorname{Sym}(\alpha) \cong \mathbb{Z}_{k}$. Some examples are

$$
t_{\operatorname{tr}\left(X^{k}\right)}=\frac{1}{k} \operatorname{tr}\left(A^{k}\right), \quad t_{\operatorname{tr}\left(X^{2} Y^{2}\right)}=\operatorname{tr}\left(A^{2} C^{2}\right), \quad t_{\operatorname{tr}\left(Y^{k}\right)}=\frac{1}{k} \operatorname{tr}\left(C^{k}\right), \quad t_{\operatorname{tr}(X Y X Y)}=\frac{1}{2} \operatorname{tr}(A C A C) .
$$

To get the dual model of the $F$ type, the techniques of Sec. II using integration in-out look inapplicable. Character expansions may work. A guess based on graph duality is 


$$
\int[d F]^{\mathbb{C}}[d G]^{\mathbb{C}} e^{-\operatorname{tr}\left(F F^{\dagger}\right)-\operatorname{tr}\left(G G^{\dagger}\right)+\sum_{k_{i},[\alpha]} s_{\left\{k_{i},[\alpha]\right\}} \operatorname{tr}\left(\alpha\left(A F B F^{\dagger}\right)^{k_{1}}\left(A F D G^{\dagger}\right)^{k_{2}}\left(C G B F^{\dagger}\right)^{k_{3}}\left(C G D G^{\dagger}\right)^{k_{4}}\right)} .
$$

Each $F$ propagator is transverse to an $X$ propagator and similarly for $G$ and $Y$. This guess has only been checked for very simple two- and three-point functions and should be treated with maximum suspicion. Here $\alpha$ is a single cycle permutation in $S_{k}$ where $k=\sum_{i=1}^{4} k_{i}$ and the coupling is defined as

$$
s_{\left\{k_{i},[\alpha]\right\}}=\frac{N}{\left|\operatorname{Sym}(\alpha) \cap \prod_{i} S_{k_{i}}\right|}
$$

[1] R. Gopakumar and C. Vafa, Adv. Theor. Math. Phys. 3, 1415 (1999).

[2] H. Ooguri and C. Vafa, Nucl. Phys. B641, 3 (2002).

[3] M. Kontsevich, Commun. Math. Phys. 147, 1 (1992).

[4] D. Gaiotto and L. Rastelli, J. High Energy Phys. 07 (2005) 053.

[5] R. Gopakumar, in Second Joburg Workshop on String Theory, 2010 (unpublished).

[6] J. M. Maldacena, Adv. Theor. Math. Phys. 2, 231 (1998); Int. J. Theor. Phys. 38, 1113 (1999); S. S. Gubser, I. R. Klebanov, and A. M. Polyakov, Phys. Lett. B 428, 105 (1998); E. Witten, Adv. Theor. Math. Phys. 2, 253 (1998).

[7] E. Brezin, V. A. Kazakov, Phys. Lett. B 236, 144 (1990).

[8] M. R. Douglas, S. H. Shenker, Nucl. Phys. B335, 635 (1990).

[9] D. J. Gross, A. A. Migdal, Phys. Rev. Lett. 64, 127 (1990).

[10] J. M. Maldacena, G. W. Moore, N. Seiberg, and D. Shih, J. High Energy Phys. 10 (2004) 020.

[11] C. Kristjansen, J. Plefka, G. W. Semenoff, and M. Staudacher,Nucl. Phys. B643, 3 (2002).

[12] N. R. Constable, D.Z. Freedman, M. Headrick, S. Minwalla, L. Motl, A. Postnikov, and W. Skiba, J. High Energy Phys. 07 (2002) 017.

[13] T. W. Brown, J. High Energy Phys. 05 (2010) 058.

[14] S. Y. Alexandrov, V. A. Kazakov, and I. K. Kostov, Nucl. Phys. B667, 90 (2003).

[15] I. R. Klebanov, arXiv:hep-th/9108019.

[16] P. H. Ginsparg and G. W. Moore, in Recent Directions in Particle Theory: From Superstrings and Black Holes to the Standard Model (TASI-92), edited by J. Harvey and J. Polchinski (World Scientific, Singapore 1993), p. 277 (Yale University Report No. YCTP-P23-92, LANL Report No. LA-UR-92-3479).

[17] D. Berenstein, J. High Energy Phys. 07 (2004) 018.

[18] A. Jevicki and T. Yoneya, J. High Energy Phys. 03 (2007) 001.

[19] S. Corley, A. Jevicki, and S. Ramgoolam, Adv. Theor. Math. Phys. 5, 809 (2002).

[20] T. S. Tai, Prog. Theor. Phys. 117, 795 (2007).

[21] L. Rastelli and M. Wijnholt, arXiv:hep-th/0507037.

[22] T. R. Morris, Nucl. Phys. B356, 703 (1991).

[23] P. Di Francesco and C. Itzykson, Annales Poincaré Phys. Theor. 59, 117 (1993).
[24] V. A. Kazakov, M. Staudacher, and T. Wynter, Commun. Math. Phys. 177, 451 (1996).

[25] V. A. Kazakov, M. Staudacher, and T. Wynter, Commun. Math. Phys. 179, 235 (1996).

[26] V. A. Kazakov, M. Staudacher, and T. Wynter, Nucl. Phys. B471, 309 (1996).

[27] V.A. Kazakov, M. Staudacher, and T. Wynter, arXiv:hep-th/9601153.

[28] R. J. Szabo and J.F. Wheater, Nucl. Phys. B491, 689 (1997).

[29] I. K. Kostov, M. Staudacher, and T. Wynter, Commun. Math. Phys. 191, 283 (1998).

[30] A. Alexandrov, A. Mironov, and A. Morozov, J. High Energy Phys. 12, (2009) 053.

[31] A. Alexandrov, arXiv:1005.5715.

[32] A. Morozov, Phys. Usp. 37, 1 (1994).

[33] A. Yu. Orlov, arxiv:math-ph/0210012v3.

[34] R. Penner, J. Diff. Geom. 27, 35 (1988).

[35] L. Chekhov and Yu. Makeenko, Phys. Lett. B 278, 271 (1992).

[36] R. Gopakumar, Phys. Rev. D 70, 025010 (2004).

[37] R. Gopakumar, Phys. Rev. D 70, 025009 (2004).

[38] R. Gopakumar, Phys. Rev. D 72, 066008 (2005).

[39] J. Ambjorn, L. Chekhov, C.F. Kristjansen, and Yu. Makeenko, Nucl. Phys. B404, 127 (1993); B449, 681(E) (1995).

[40] D.E. Berenstein, J. M. Maldacena, and H. S. Nastase, J. High Energy Phys. 04 (2002) 013.

[41] C. Imbimbo and S. Mukhi, Nucl. Phys. B449, 553 (1995).

[42] R. Dijkgraaf, G. W. Moore, and R. Plesser, Nucl. Phys. B394, 356 (1993).

[43] S. Mukhi, arXiv:hep-th/0310287.

[44] A. Mukherjee and S. Mukhi, J. High Energy Phys. 10 (2005) 099.

[45] M.L. Mehta, Random matrices (Academic Press, New York, 1991).

[46] I. R. Klebanov and D. A. Lowe, Nucl. Phys. B363, 543 (1991).

[47] V. Fateev, A. Zamolodchikov, and Al. Zamolodchikov, arXiv:hep-th/0001012; J. Teschner, arXiv:hep-th/ 0009138; 
[48] J. M. Maldacena, J. High Energy Phys. 09 (2005) 078; Int. J. Geom. Methods Mod. Phys. 3, 1 (2006)

[49] A. Zamolodchikov and Al. Zamolodchikov, arXiv:hep-th/ 0101152.

[50] V. Balasubramanian, M. Berkooz, A. Naqvi, and M.J. Strassler, J. High Energy Phys. 04 (2002) 034.

[51] O. Ganor, J. Sonnenschein, and S. Yankielowicz, Nucl. Phys. B434, 139 (1995).

[52] S. Cordes, G. W. Moore, and S. Ramgoolam, Nucl. Phys. B, Proc. Suppl. 41, 184 (1995).

[53] C. Itzykson and J. B. Zuber, J. Math. Phys. (N.Y.) 21, 411 (1980).

[54] D. Vaman and H. L. Verlinde, J. High Energy Phys. 11 (2003) 041.

[55] A. Mironov, A. Morozov, and S. Natanzon, Theor. Math. Phys. 166, 1 (2011).

[56] R.d. M. Koch and S. Ramgoolam, arXiv:1002.1634.

[57] G. V. Belyi, Mathematics of the USSR Izvestija 14, 247 (1980).

[58] R. Pandharipande, arXiv:math. AG/9912166.

[59] A. Okounkov, arXiv:math. AG/0004128.

[60] A. Okounkov and R. Pandharipande, arXiv:math/0204305.

[61] M. Aganagic, R. Dijkgraaf, A. Klemm, M. Marino, and C. Vafa, Commun. Math. Phys. 261, 451 (2006).

[62] K. Strebel, Quadratic Differentials (Springer-Verlag, Berlin, 1984).
[63] J. L. Harer, in Theory of Moduli, Montecatini Terme, 1985, edited by Edoardo Sernesi (Springer-Verlag, Berlin, 1988), p. 138 .

[64] E. Witten, Nucl. Phys. B340, 281 (1990).

[65] R. Dijkgraaf, arXiv:hep-th/9201003.

[66] O. Aharony, Z. Komargodski, and S. S. Razamat, J. High Energy Phys. 05 (2006) 016.

[67] O. Aharony, J. R. David, R. Gopakumar, Z. Komargodski, and S. S. Razamat, Phys. Rev. D 75, 106006 (2007).

[68] S. S. Razamat, J. High Energy Phys. 07 (2008) 026.

[69] S. S. Razamat, J. High Energy Phys. 03 (2010) 049.

[70] V. A. Kazakov, Nucl. Phys. B587, 645 (2000).

[71] E. Witten, Phys. Rev. D 44, 314 (1991).

[72] S. Mukhi and C. Vafa, Nucl. Phys. B407, 667 (1993).

[73] O. Aharony, O. Ganor, J. Sonnenschein, and S. Yankielowicz, Phys. Lett. B 305, 35 (1993).

[74] C. Candu, T. Creutzig, V. Mitev, and V. Schomerus, J. High Energy Phys. 05 (2010) 047.

[75] D. Ghoshal, S. Mukhi, and S. Murthy, J. High Energy Phys. 11 (2004) 027.

[76] I. Ellwood and A. Hashimoto, J. High Energy Phys. 02 (2006) 002.

[77] I. K. Kostov, Nucl. Phys. B575, 513 (2000).

[78] E. Witten, Nucl. Phys. B371, 191 (1992).

[79] M. Aganagic, H. Ooguri, N. Saulina, and C. Vafa, Nucl. Phys. B715, 304 (2005). 